Research Article

\title{
Synchronous Generator Excitation System for a Ship Based on Active Disturbance Rejection Control
}

\author{
Rongjie Wang, ${ }^{1,2}$ Xiangyu Liu, ${ }^{1}$ and Yuyuan Huang $\mathbb{D}^{1,2}$ \\ ${ }^{1}$ Marine Engineering Institute, Jimei University, Xiamen 361021, China \\ ${ }^{2}$ Fujian Provincial Key Laboratory of Naval Architecture and Ocean Engineering, Xiamen 361021, China \\ Correspondence should be addressed to Yuyuan Huang; yyhuang2504@163.com
}

Received 17 December 2020; Revised 24 March 2021; Accepted 7 April 2021; Published 28 April 2021

Academic Editor: Qiuye Sun

Copyright ( 2021 Rongjie Wang et al. This is an open access article distributed under the Creative Commons Attribution License, which permits unrestricted use, distribution, and reproduction in any medium, provided the original work is properly cited.

To solve synchronous generator oscillations in marine power systems which cannot be effectively suppressed, according to the nonlinearity and time variability of the ship power system, a method of synchronous generator excitation control for a ship based on active disturbance rejection control (ADRC) is proposed. Under different working conditions, three methods are automatic voltage regulator (AVR), automatic voltage regulator with power system stabilizer (PSS), and ADRC methods, which are applied to the two-generator parallel-running excitation system of a ship in simulations. The simulation results show that the excitation control system based on ADRC is faster and has better anti-interference ability and has a better restraining effect on synchronous generator oscillation.

\section{Introduction}

A ship power system is an islanded power system. When the running conditions change or the load changes suddenly, due to the inertial action of the synchronous generator rotor, the power angle cannot be instantaneously maintained at a new stable value, and adjustment of the near the steady-state value under the action of the excitation control system is required; that is, synchronous generator oscillation occurs $[1,2]$. This phenomenon reduces the stability of the synchronous generator set, and generator instability can occur, thereby affecting the safe and stable running of the entire ship power system [3]. Therefore, the generator excitation system of a ship has always been a popular research topic. To restrain synchronous generator oscillation, a generator excitation system in the " $X+$ AVR (automatic voltage regulator)" mode, where " $\mathrm{X}$ " is the control method, is used on land. At present, the studies that have been carried out on control methods mainly focus on single variable control, multivariable control, and nonlinear control [4-13]. However, the generator excitation system in the " $X+$ AVR" mode has shortcomings such as unreasonable error examples; difficulty with realizing differential signals, which must be improved; and unreasonable linear combinations of error signals, which cannot resolve the contradiction between rapidity and overshooting. In connection with the synchronous generator oscillation of a ship power system, an excitation system in the "AVR + PSS (power system stabilizer)" mode is mainly used $[14,15]$. When the disturbance it is subjected to is relatively small, the system can maintain approximate linearization characteristics; however, when the disturbance the system is subjected to is relatively large, leading to deviation from the normal working point, the approximate linearization characteristics cannot be maintained, thereby leading to a decrease in the performance of the control system. Due to the unique nature of the running of the ship power system, the existing excitation system in the " $X+\mathrm{AVR}$ " and "AVR + PSS" modes presents a negative damping effect $[16,17]$; thus, synchronous generator oscillations cannot be effectively restrained. The active disturbance rejection control (ADRC) in [18] regards the internal and external disturbances of the system as a total disturbance. ADRC combines the advantages of modern control theory and nonlinear theory [19] and has a good control effect even for nonlinear systems that are difficult to establish accurate mathematical models. Because the 
algorithm does not depend on the specific model of the control object [20], it has the advantages of high control accuracy and strong anti-interference ability, so it has been widely used in important fields such as national defense and scientific research [21]. The total disturbance of the system is estimated in real time through an extended state observer, and then the feedback loop of the system is quickly compensated in with this estimated value of the total disturbance, which is a control method suitable for use in nonlinear systems and is based on the proportional-integralderivative principle [22, 23]. Therefore, this paper attempts to apply ADRC to the synchronous generator excitation system of a ship from the perspective of compensating the damping of the AVR for the ship power system.

\section{Analysis of the Negative Damping Effect of the Synchronous Generator Oscillation of a Ship Power System}

When the generator is disturbed in the running process, deviations are generated in its various electrical parameters. The electromagnetic torque, no-load electromotive force, generator voltage, and rotor angle of the synchronous generator are recorded as $M_{e}, E_{q}, U_{t}$, and $\delta$, respectively, and the corresponding amounts of change are $\Delta M_{e}, \Delta E_{q}, \Delta U_{t}$, and $\Delta \delta$, respectively. Their Heffron-Phillips model is expressed in

$$
\begin{aligned}
\Delta M_{\mathrm{e}} & =K_{1} \Delta \delta+K_{2} \Delta E_{q}^{\prime}, \\
\Delta E_{q}^{\prime} & =\frac{K_{3}}{1+T_{d 0}^{\prime} K_{3} s} \Delta E_{f d}-\frac{K_{3} K_{4}}{1+T_{d 0}^{\prime} K_{3} s} \Delta \delta, \\
\Delta U_{t} & =K_{5} \Delta \delta+K_{6} \Delta E_{q}^{\prime}, \\
\Delta \delta & =\frac{\omega_{0}}{T_{J} s^{2}}\left(\Delta M_{\mathrm{m}}-\Delta M_{e}\right) .
\end{aligned}
$$

The structural block diagram corresponding to equation (1) is shown in Figure 1.

As seen in Figure 1, the excitation system changes $\Delta M_{e 2}$ by changing $\Delta E_{q}^{\prime}$. The transfer function of the entire openloop system is obtained by moving the $K_{4}$ summing point forward:

$$
\frac{\Delta M_{e 2}(s)}{\Delta \delta(s)}=\frac{K_{2} G_{3}\left(K_{4}+K_{5} G_{E}\right)}{1+G_{E} G_{3} K_{6}} .
$$

Then,

$$
G_{3}=\frac{K_{3}}{1+K_{3} T_{d 0}^{\prime} s} .
$$
is

For the rapid excitation system, the transfer function $G_{E}$

$$
G_{E}(s)=\frac{K_{A}}{1+T_{E} s} .
$$

Substituting equations (3) and (4) into equation (2), we can obtain

$$
\frac{\Delta M_{e 2}}{\Delta \delta}=-\frac{K_{2} K_{3} K_{4}\left(1+T_{E} s\right)+K_{2} K_{3} K_{5} K_{A}}{\left(1+K_{3} T_{d 0}^{\prime} s\right)\left(1+T_{E} s\right)+K_{3} K_{6} K_{A}} .
$$

Since in the rapid excitation system, $T_{E} \approx 0, K_{3} K_{6} K_{\mathrm{A}}$ is far larger than 1, so we let $s=j \omega_{d}$. Equation (5) is simplified to obtain

$$
\frac{\Delta M_{e 2}}{\Delta \delta}=\frac{\left(K_{2} / K_{6}\right)\left(K_{4} / K_{A}+K_{5}\right)}{1+j \omega_{d} T_{d 0}^{\prime} / K_{A} K_{6}} .
$$

Setting $T_{\mathrm{EQ}}=T_{d 0}^{\prime} /\left(K_{\mathrm{A}} K_{6}\right)$, we have

$$
\frac{\Delta M_{e 2}}{\Delta \delta}=\frac{K_{2}\left(K_{4} / K_{A}+K_{5}\right)}{K_{6}\left(1+\omega^{2} T_{E Q}^{2}\right)}+j \omega_{d} \frac{K_{2} T_{E Q}\left(K_{4} / K A+K_{5}\right)}{K_{6}\left(1+\omega^{2} T_{E Q}^{2}\right)} \text {. }
$$

Equation (7) can be rewritten as

$$
\Delta M_{e 2}=\Delta M_{s} \Delta \delta+\Delta M_{d} s \Delta \delta .
$$

Equation (8) shows that two torque components can be generated due to flux linkage changes, that is, the synchronous torque $\Delta M_{s} \Delta \delta$ and the damping torque $\Delta M_{D} s \delta$, where

$$
\begin{aligned}
& \Delta M_{s}=\frac{K_{2}\left(K_{4} / K_{A}+K_{5}\right)}{K_{6}\left(1+\omega^{2} T_{E Q}^{2}\right)} \approx \frac{K_{2} K_{5}}{K_{6}\left(1+\omega^{2} T_{E Q}^{2}\right)}, \\
& \Delta M_{d}=\frac{K_{2} T_{E Q}\left(K_{4} / K_{A}+K_{5}\right)}{K_{6}\left(1+\omega^{2} T_{E Q}^{2}\right)} \approx \frac{K_{2} K_{5} T_{E Q}}{K_{6}\left(1+\omega^{2} T_{E Q}^{2}\right)} .
\end{aligned}
$$

Considering the inherent damping torque $D_{S} \Delta \delta$ and the synchronous torque $K_{1} \Delta \delta$ of the synchronous generator, the conditions necessary for oscillation instability and nonperiodic instability not to occur to the synchronous generator are expressed in

$$
\begin{aligned}
D+\Delta M_{d} & >0, \\
K_{1}+\Delta M_{s} & >0 .
\end{aligned}
$$

The ship power system is often in a state of heavy load. In this case, $\delta$ is relatively large, and $K_{5}<0$. From equation (9), $\Delta M_{\mathrm{s}}>0$ is obtained; that is, the AVR causes the synchronous ability of the system to increase; from equation (10), $\Delta M_{\mathrm{D}}<0$ is obtained; that is, the AVR causes the total damping torque of the system to decrease. With increasing voltage amplification coefficient $K_{\mathrm{A}}, T_{\mathrm{EQ}}$ decreases, and $\left|\Delta M_{\mathrm{D}}\right|$ increases. Because the AVR uses voltage as the control variable, the phase of the additional flux linkage it provides lags behind $\Delta \delta$ and has a component that is a reversed-phase quantity with respect to $\Delta \omega$. When the negative damping torque it generates causes the total damping torque coefficient to satisfy $D+\Delta M_{\mathrm{D}}<0, \Delta \delta$ becomes large, leading to a decrease in the stability of the system, and it is impossible to effectively restrain the oscillation phenomenon. This type of phenomenon in which the AVR worsens the damping of the generator set can be expressed in Figure 2.

When the system is in an equilibrium state, because it is subjected to external disturbance, the $\delta$ angle generates 


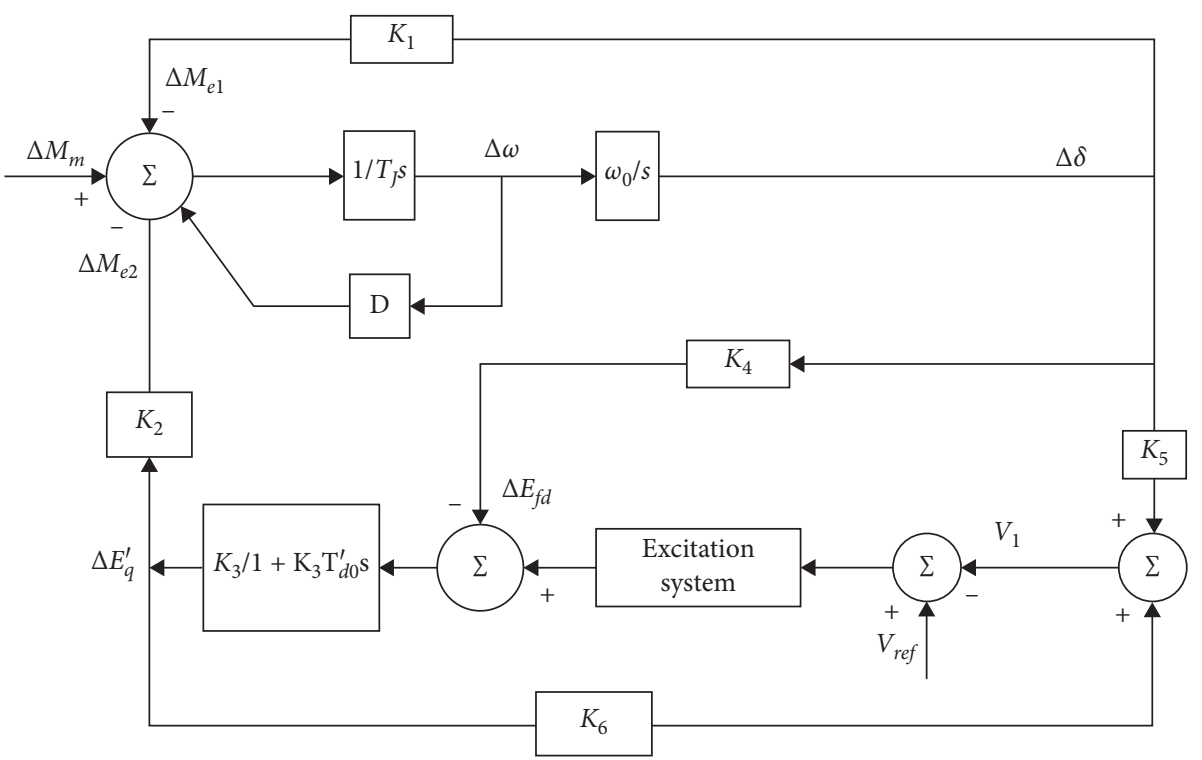

FIGURE 1: Heffron-Phillips model of the synchronous generator.

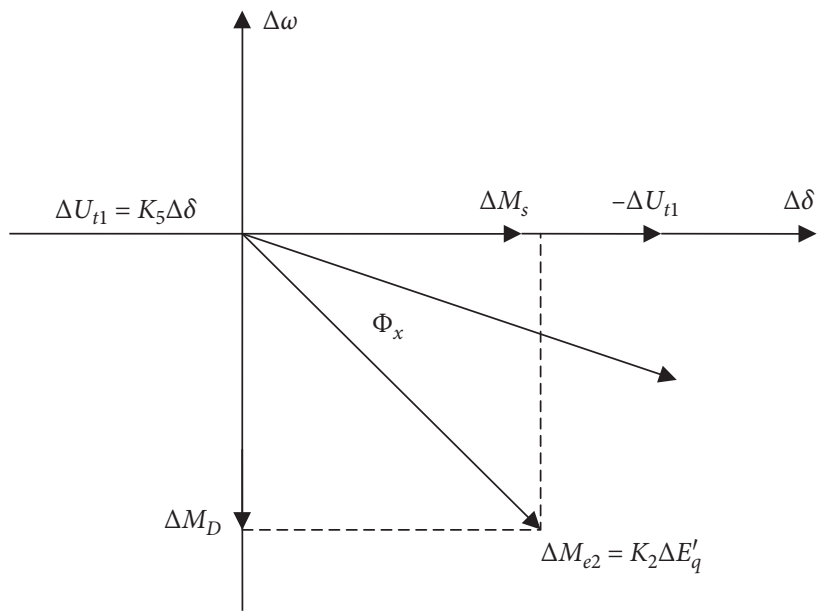

FIgURE 2: Torque vector diagram.

oscillations, which is expressed as $\Delta \delta$. Let $K_{5}<0$; then the first component of the voltage deviation $\Delta U_{\mathrm{t} 1}$ is the reverse of $\Delta \delta$. Afterwards, $\Delta E_{f d}$ is input into the excitation system, and the output $\Delta E_{\mathrm{q}}{ }_{\mathrm{q}}$ is proportional to $\Delta M_{\mathrm{e} 2}$. When the AVR amplification multiple is relatively large, $K_{4}$ can be ignored, as it is approximately equal to 0 . Then, the block diagram of the excitation system control is shown in Figure 3. The transfer function of Figure 3 is

$$
\frac{\Delta M_{e 2}}{\Delta U_{t 1}}=-\frac{K_{2} K_{3} K_{A}}{K_{3} K_{6} K_{A}+\left(1+K_{3} T_{d 0}^{\prime} s\right)\left(1+T_{E} s\right)} .
$$

Because $T_{\mathrm{E}}<<1$ and $K_{3} K_{6} K_{\mathrm{A}}>>1$, equation (13) is simplified as

$$
\frac{\Delta M_{e 2}}{\Delta U_{t 1}}=-\frac{K_{2} / K_{6}}{1+T_{d 0}^{\prime} / K_{6} K_{A} s}
$$

As seen from equation (14), the part from $-\Delta U_{\mathrm{t} 1}$ to $\Delta M_{\mathrm{e} 2}$ is equivalent to an inertia link, which generates a phase lag of $\Phi_{x}=0-90^{\circ}$. Figure 2 shows that the component of $\Delta M_{\mathrm{e} 2}$ on the $\Delta \omega$ axis is a negative value; that is, the damping torque is negative. In the oscillation process, if $\delta$ increases, the terminal voltage $U_{\mathrm{t} 1}$ decreases, and the excitation voltage is increased after AVR regulation; however, due to the inertial action, the flux linkage still increases when the rotational speed $\Delta \omega$ is a negative value, which causes the electromagnetic torque of braking to increase; that is, the so-called "negative damping" effect. If a device is added to the excitation, it can generate a positive damping torque large enough to make the position of the combined torque of this torque with $\Delta M_{\mathrm{e} 2}$ positive, which can compensate for the phase lag of the excitation system and restrain the oscillation of the system. 


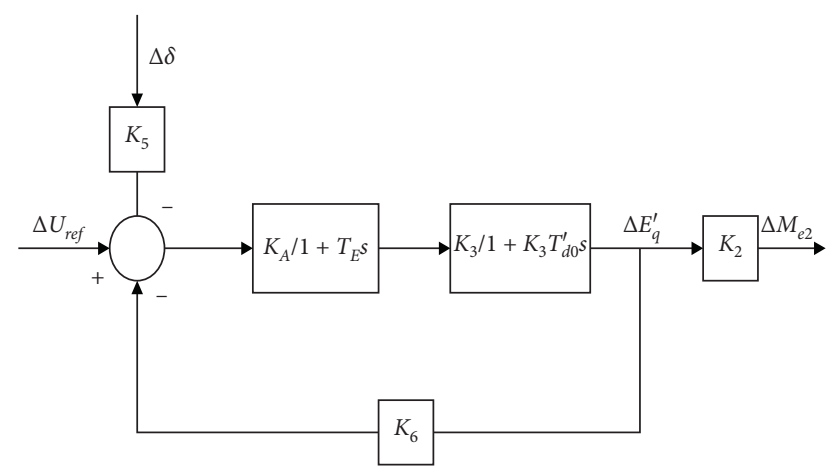

FIGURE 3: Block diagram of the generator excitation system that ignores $\mathrm{K}_{4}$.

\section{ADRC-Based Control Methods for the Synchronous Generator Excitation System of a Ship}

3.1. Principle of the Active Disturbance Rejection Controller. The ADRC is mainly composed of three parts, the tracking differentiator (TD), the extended state observer (ESO), and the nonlinear state error feedback (NLSEF) control law. Its structural block diagram is shown in Figure 4. $v(t)$ in Figure 4 is the system input signal. After $v(t)$ enters the TD, the TD carries out rapid tracking of $v(t)$ and obtains the two output signals $v_{1}$ and $v_{2}$, where $v_{1}$ is the tracking signal of $v(t)$ and $v_{2}$ is the differential signal of $v(t)$. The output signal of ESO is the output signal of the controlled object, and three output signals $-z_{1}, z_{2}$, and $z_{3}$-are given after state estimation. $z_{1}$ and $z_{2}$ are the estimated values of the state variables by the ESO for an uncertain model. $z_{3}$ is the total disturbance estimate by the ESO for an uncertain model. Afterwards, $v_{1}$ and $z_{1}$ as well as $v_{2}$ and $z_{2}$ are compared to obtain two sets of error signals, $e_{1}$ and $e_{2}$. NLSEF carries out nonlinear combination of the two sets of error signals to obtain the control signal $u_{0}(t)$; the control signal is multiplied by the ESO-estimated total disturbance state variable $z_{3}$ and the coefficient $1 / b$, and the final control value $u(t)$ is obtained.

3.2. Design of the Active Disturbance Rejection Controller for the Synchronous Generator Excitation System of a Ship. Each part of the ADRC can be independently designed according to the "separation principle" and then combined into a complete closed-loop controller. The steps for the design of the active disturbance rejection controller for the synchronous generator excitation system of a ship are as follows.

3.2.1. Modeling of the Controlled Object. The ADRC controller does not require an accurate mathematical model; an approximate model suffices. The approximate mathematical model of the synchronous generator excitation system is

$$
\left\{\begin{array}{l}
\dot{\delta}=\omega-\omega_{0}, \\
\dot{\omega}=\frac{\omega}{H}\left(P_{\mathrm{m}}-P_{\mathrm{e}}\right)-\frac{D}{H}\left(\omega-\omega_{0}\right) \\
E_{\mathrm{q}}^{\prime}=-\frac{1}{T_{\mathrm{d} 0}} E_{\mathrm{q}}+\frac{1}{T_{\mathrm{d} 0}} E_{f} .
\end{array}\right.
$$

3.2.2. Arranging the Transition Process. The purpose of arranging the transition process is to quickly and accurately track the input signal and the differential of the input signal. In this paper, the terminal voltage rating taken by the ADRC for the synchronous generator is the TD input voltage $v$, and the fastest comprehensive function of the transition process is fhan. The TD transition process is arranged as

$$
\left\{\begin{array}{l}
\dot{v}_{1}=v_{1}+h v_{2}, \\
\dot{v}_{2}=v_{2}+h \operatorname{fhan}\left(v_{1}-v_{2}, r, h\right),
\end{array}\right.
$$

where $r$ is an adjustable parameter and $h$ is the integration step size.

3.2.3. Design of the ESO. The functional relationship between the terminal voltage and the excitation voltage of the synchronous generator is regarded as an unknown function $f(x)$; then the state spatial equation of the excitation system can be expressed as

$$
\left\{\begin{array}{l}
\dot{x}_{1}=x_{2}, \\
\dot{x}_{2}=f\left(x_{1}, x_{2}\right)+b u, \\
y=x_{1} .
\end{array}\right.
$$

Expanding $f\left(x_{1}, x_{2}\right)$ into a new state variable, we have

$$
\left\{\begin{array}{l}
\dot{x}_{1}=x_{2}, \\
\dot{x}_{2}=x_{3}+b u, \\
\dot{x}_{3}=w \\
y=x_{1} .
\end{array}\right.
$$




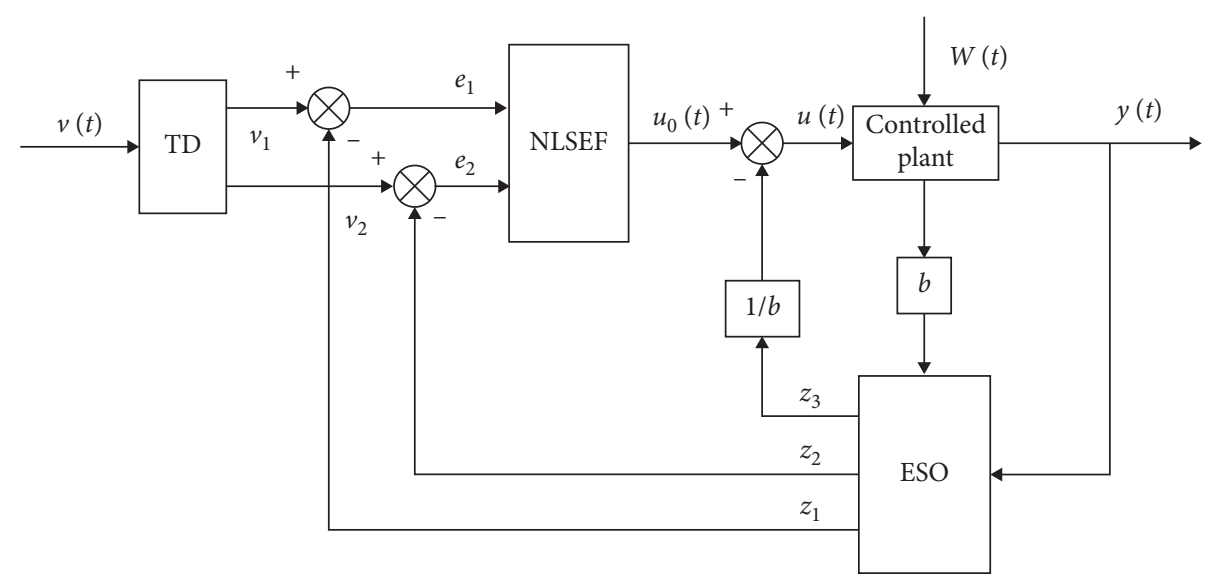

FIgURE 4: Structural block diagram of the active disturbance rejection controller.

By introducing the fal function, we can design the following third-order state observer. Through this state observer, the total disturbance of the excitation system and the terminal voltage $U_{t}$ of the synchronous generator are estimated:

$$
\left\{\begin{array}{l}
e=z_{1}-y_{1}, \\
z_{1}=z_{1}+h\left(z_{2}-\beta_{01} e-u_{1}\right), \\
z_{2}=z_{2}-h \beta_{02}-h f a l\left(e, \alpha_{1}, h\right), \\
z_{3}=z_{3}-h \beta_{03} f a l\left(e, \alpha_{2}, \delta\right),
\end{array}\right.
$$

where $\beta_{01}, \beta_{02}$, and $\beta_{03}$ are adjustable parameters and $h$ is the integration step size.

3.2.4. Design of the Nonlinear Error Feedback. The design of the nonlinear error feedback is shown in the following equation:

$$
\left\{\begin{array}{l}
e_{1}=v_{1}-z_{1}, \\
e_{2}=v_{2}-z_{2}, \\
u_{0}=k_{1} f a l\left(e_{1}, \alpha_{1}, \delta\right)+k_{2} f a l\left(e_{2}, \alpha_{2}, \delta\right), \\
u=u_{0}-\frac{z_{3}}{b},
\end{array}\right.
$$

where $u$ is the control variable $U_{\mathrm{f}}, b$ is the control variable coefficient, and $k_{1}$ and $k_{2}$ are adjustable parameters.

After the three core links of ADRC, that is, the TD, the ESO, and the nonlinear error feedback, have been designed, they are connected in accordance with the input of the controlled object, the output, and the input and output of each module according to the logical relationship, and an ADRC-based synchronous generator excitation control system for a ship is constructed. The structural block diagram of control is shown in Figure 5.

Figure 5 shows that the system input is the reference

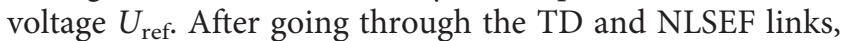
the initial control voltage $u_{0}(t)$ is output. Afterwards, the
ESO carries out disturbance compensation for $-z 3 / b$ to obtain the control voltage $u(t)$. The control voltage $u(t)$ undergoes limiting, and the excitation voltage $U_{\mathrm{f}}$ is output after the exciter starts the excitation. Afterwards, the voltage of the synchronous generator is measured to obtain the output terminal voltage $U_{\mathrm{t}}$, and it is fed back to the ESO to complete closed-loop control.

3.2.5. Parameter Tuning Methods. There are currently no specific methods for ADRC parameter tuning. Manual adjustment of parameters can be carried out only according to empirical methods. In this paper, parameter adjustment is carried out according to the order of TD, ESO, and NLSEF, and actual simulation experience is combined with data from relevant literature. The following is a summary of the experience in ADRC parameter tuning.

According to the TD principle described in the above text, the TD is a system that contains linear and nonlinear parts, and the selection of its nonlinear function and parameters greatly affects the performance of the TD. The main parameters of the TD are $r$ and $h$. According to experience, $r$ mainly determines the speed in tracking the signal, and $h$ mainly affects the filtering effect of the TD and the quality of the differential signal. The parameter $r$ cannot take on an overly large value to ensure the tracking speed; otherwise, the quality of the output signal is reduced, the TD output signal is the same as the input signal, the function of arranging the transition process is lost, and the contradiction between speed and overshooting cannot be resolved. The parameter $h$ should not take on an overly small value; otherwise, the quality of the differential signal cannot be ensured. This parameter is generally between 0.001 and 0.01 .

The role of the ESO as the core of ADRC is to accurately estimate the state variables and disturbances and compensate for the control signal $u_{0}$. If the parameters of the ESO are not regulated properly, the entire closed-loop control is greatly affected. $\beta_{01}, \beta_{02}$, and $\beta_{03}$ are the three main parameters of the ESO. A large amount of simulation experience and data show the following. The larger $\beta_{01}$ is, the faster $z_{1}$ tracks a given signal $y$ and the faster $z_{2}$ tracks the derivative of $y$. However, when $\beta_{01}$ is too large, an oscillation 


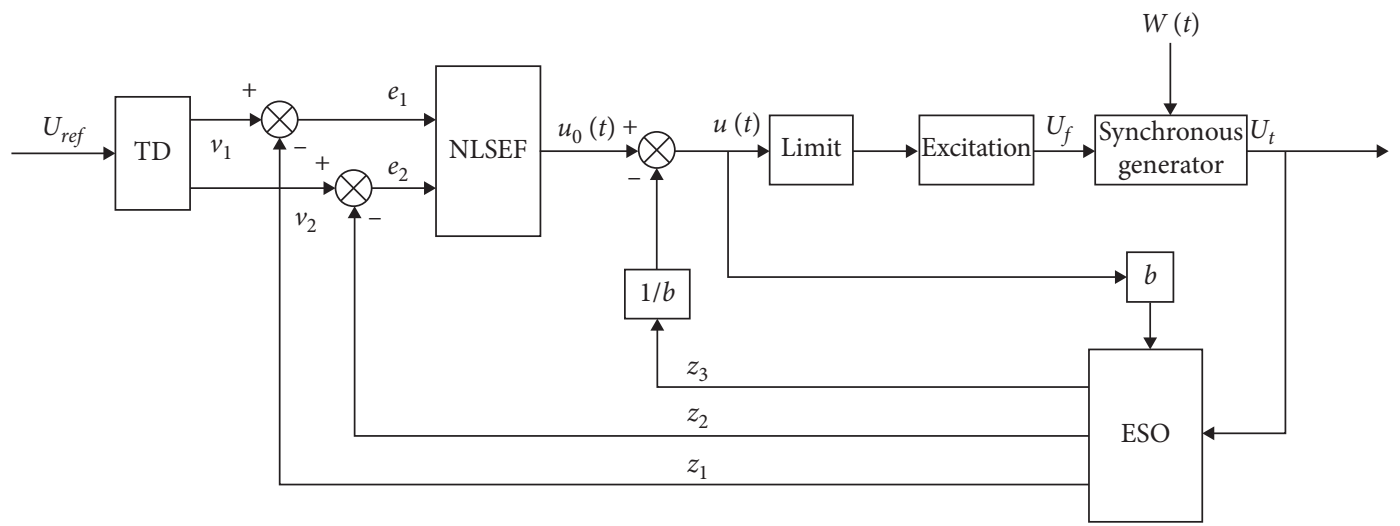

FIGURE 5: Structural block diagram of the ADRC-based synchronous generator excitation control system of a ship.

phenomenon appears in $z_{2}$ and $z_{3}$; when $\beta_{01}$ is too small, a divergence phenomenon appears in the three state variables. The parameter $\beta_{02}$ does not have too great of an effect on the state variable $x_{1}$; with increasing $\beta_{02}$, the estimation effect of ESO on the disturbance $x_{3}$ worsens, and high-frequency noise appears, leading to a failure to track the disturbance; if $\beta_{02}$ is too small, then a divergence phenomenon appears in $z_{1}$ and $z_{2}$. The parameter $\beta_{03}$ has a greater effect on $z_{1}$ and $z_{2}$, and an increase in the value of $\beta_{03}$ causes $z_{1}$ and $z_{2}$ to diverge. Decreasing the value of $\beta_{03}$ generates a large error in the estimated value of $x_{1} z_{1}$, and the estimated value of the disturbance $z_{3}$ becomes very poor. We can see from the above experience that, only by fully considering the constraint relationship between the three parameters of $\beta_{01}, \beta_{02}$, and $\beta_{03}$ can a more ideal simulation result be obtained.

According to the principle of nonlinear state error feedback, the role of the two parameters $k_{1}$ and $k_{2}$ is similar to that of $k_{\mathrm{p}}$ and $k_{\mathrm{d}}$ in proportional-integral-derivative (PID) control. Increasing the parameter $k_{1}$ can improve the rapidity of system regulation, but the amount of overshooting increases; the rapidity of the system is reduced by decreasing the parameter $k_{1}$, and the amount of overshooting decreases. The role of the parameter $k_{2}$ is opposite to that of $k_{1}$; increasing $k_{2}$ decreases the amount of overshooting, and the system regulation time increases. Decreasing $k_{2}$ increases the speed of the system and the amount of overshooting. Changes in the parameter $b$ greatly change the control variable $u$. As seen from the logical relationships in Figures 5-8, increasing $b$ can increase the control variable $u$, but decreasing $b$ decreases the compensation for the disturbance. Selecting an appropriate value for the parameter $b$ is very important for the disturbance compensation effect.

\section{Simulation Verification}

4.1. ADRC Simulation Model. First, the simulation models for the three core parts-TD, ESO, and NLSEF-are established.

4.1.1. TD Simulation Model. The TD simulation model established in accordance with equation (16) is shown in Figure 6.

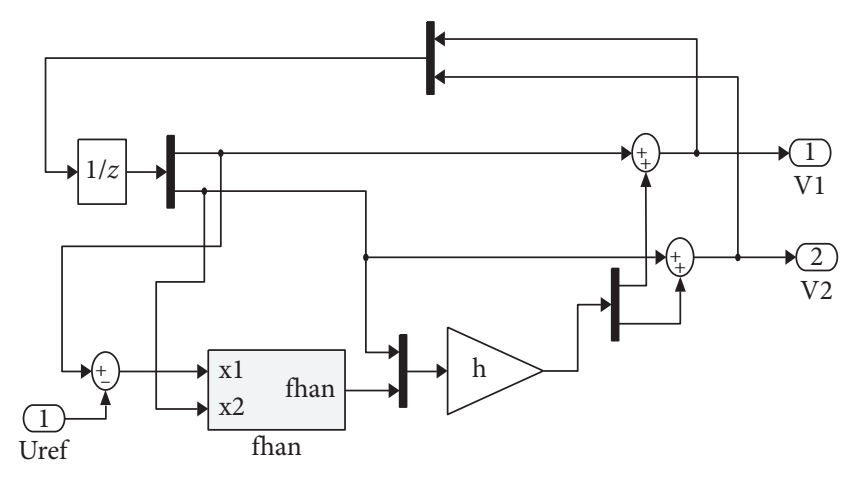

Figure 6: TD simulation model.

To verify the effectiveness of the TD simulation model built, a simple verification simulation is carried out. Given the input of a sinusoidal signal of $\sin (t)$, the initial input signal of the TD is $v(t)=\sin (\mathrm{t})$. The simulation parameter settings are an integration step size $h=0.005$ and $r=100$. The simulation curves are shown in Figure 7.

The TD parameters are changed to an integration step size $h=0.005$ and $r=200$, and the simulation curves are shown in Figure 8.

The TD parameters are changed to an integration step size $h=0.008$ and $r=100$, and the simulation curves are shown in Figure 9.

As seen from the above three simulation diagrams, the established TD simulation model has a relatively good tracking effect on the input signal and can obtain a goodquality differential signal. Comparison of Figures 7 and 8 shows that when the value of the parameter $r$ is increased, the signal tracking is better, but the fluctuations in the differential signal increase. Comparison of Figures 8 and 9 shows that increasing the value of $h$ can obviously improve the tracking result but reduces the quality of the differential signal.

4.1.2. ESO Simulation Model. The established ESO simulation model is shown in Figure 10.

In the figure, the two fal modules are $m$ files written with the S-function. 


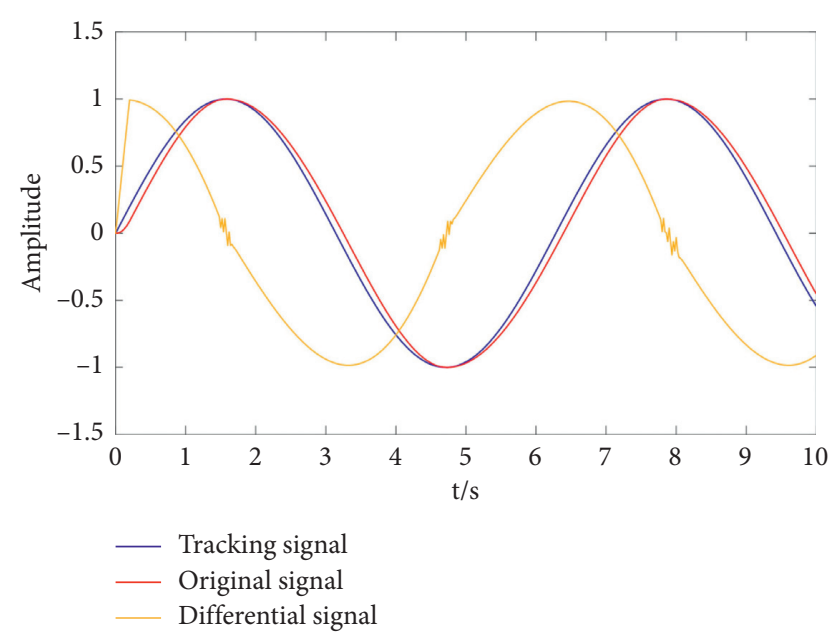

FIGURE 7: Simulation curves with $r=100$ and $h=0.005$.

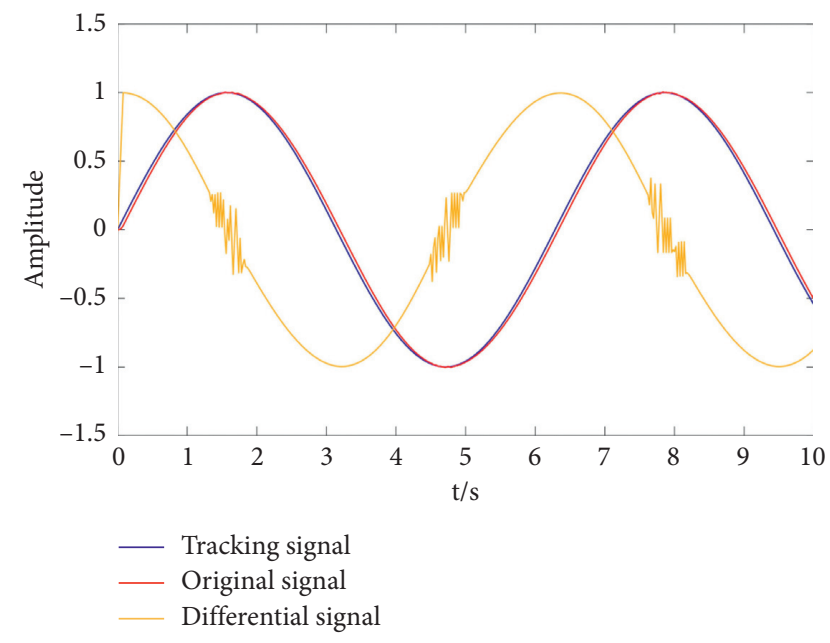

FIGURE 8: Simulation curves with $r=200$ and $h=0.005$.

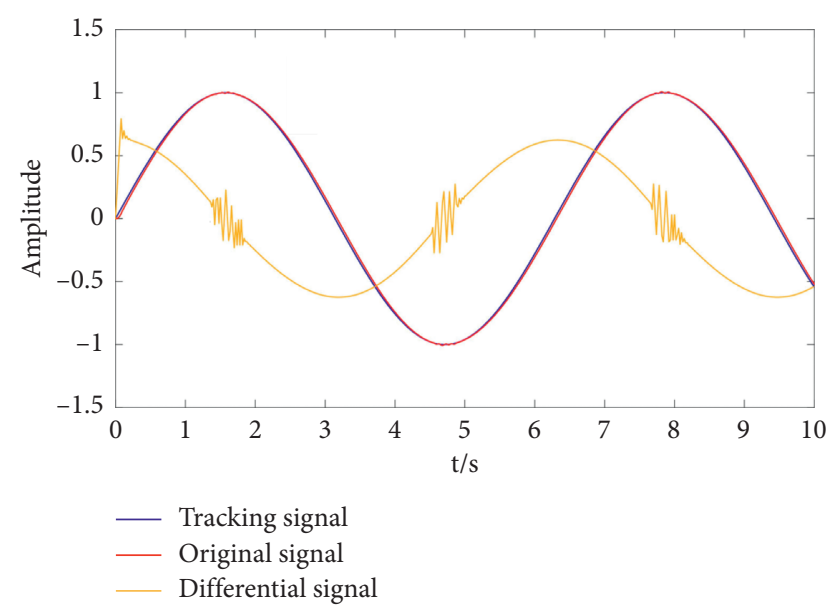

FIGURE 9: Simulation curves with $r=100$ and $h=0.008$.
4.1.3. NLSEF Simulation Model. According to equation (20), the $m$ files are written with the S-function. The established NLSEF simulation model is shown in Figure 11.

In Figure 11, the initial control variable $u$ can be output by carrying out nonlinear feedback regulation on the two errors of $e_{1}$ and $e_{2}$ and carrying out reasonable parameter regulation.

The simulation models of the above three modules are packaged and connected to form a complete ADRC simulation model, shown in Figure 12.

To verify the effectiveness of the established ADRC simulation model, a simple verification simulation experiment is carried out. The controlled object is assumed to be

$$
G(s)=\frac{1}{s^{2}+3 s+1} .
$$

The simulation parameter settings are as follows: TD parameters $r=100$ and $h=0.005$; ESO parameters $\beta_{01}=50$, $\beta_{02}=300$, and $\beta_{03}=10$; compensation coefficient $b=0.5$; nonlinear state feedback parameters $\alpha_{1}=0.5, \alpha_{2}=0.75$, and $\delta=0.1$. The TD input is a sinusoidal signal, and the simulation curve is shown in Figure 13.

The above simulation curve shows that the establishment of the ADRC controller simulation model is successful. Moreover, Figure 13 shows that the ESO can very accurately estimate state $x_{1}$. Figure 14 shows that the ESO can carry out more accurate estimation for state $x_{2}$ but that the estimation is not as good as the estimation for $x_{1}$, which may be related to the settings for the three parameters of $\beta_{01}, \beta_{02}$, and $\beta_{03}$.

4.2. Sudden Loading and Sudden Unloading Simulation Test. The ADRC simulation model in Figure 12 and the twogenerator parallel-running simulation model of a ship power system are combined to establish an ADRC-based twogenerator parallel-running simulation model of a ship power system. The simulation model is shown in Figure 15.

To verify the effectiveness of the ADRC-based twogenerator parallel-running simulation model of a ship power system established in Figure 15 and to verify the restraining effect of the ADRC-based synchronous generator excitation control system of a ship on the synchronous generator oscillation shown in Figure 15, the three excitation control methods (AVR, AVR + PSS2B, and ADRC) are used to carry out simulation experiments under different working conditions to verify the effectiveness of the proposed method.

The following parameters of the synchronous generator from a certain real ship are used. The parameters of the synchronous generators $\mathrm{G} 1$ and $\mathrm{G} 2$ are as follows: $P_{\mathrm{n}}=3.125 \times 10^{6}$ VA, $U_{\mathrm{n}}=450 \mathrm{~V}, f_{\mathrm{n}}=60 \mathrm{~Hz}, X_{\mathrm{d}}=1.56$, $X_{\mathrm{d}}{ }^{\prime}=0.296, X_{\mathrm{d}}{ }^{\prime}=0.177, X_{\mathrm{q}}{ }^{\prime}=1.06, X_{\mathrm{q}}{ }^{\prime \prime}=0.177, X_{1}=0.052$, $T_{\mathrm{d}}{ }^{\prime}=3.7, T_{\mathrm{d}}{ }^{\prime \prime}=0.05$, and $T_{\mathrm{q} 0}=0.05$. The parameters of the TD are as follows: $r=100, h=0.005$; those of ESO are as follows: $\beta_{01}=80, \beta_{02}=50, \beta_{03}=10, h=0.1, \alpha_{1}=0.75$, and $\alpha_{2}=0.75$; and those of the nonlinear state feedback are as follows: $k_{1}=35, k_{2}=150, \alpha_{1}=0.5$, and $\alpha_{2}=1.25$. 


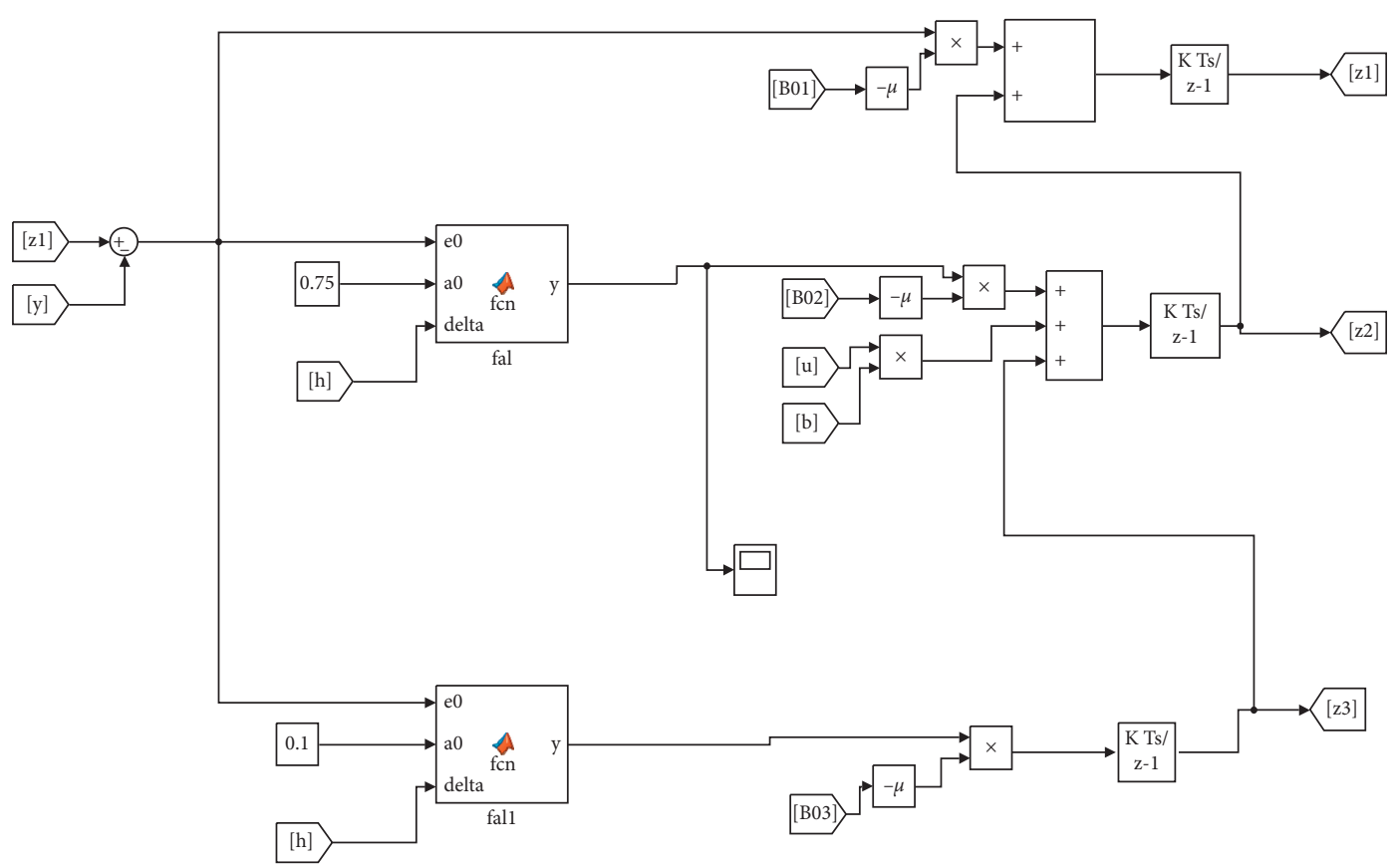

FIGURE 10: ESO simulation model.

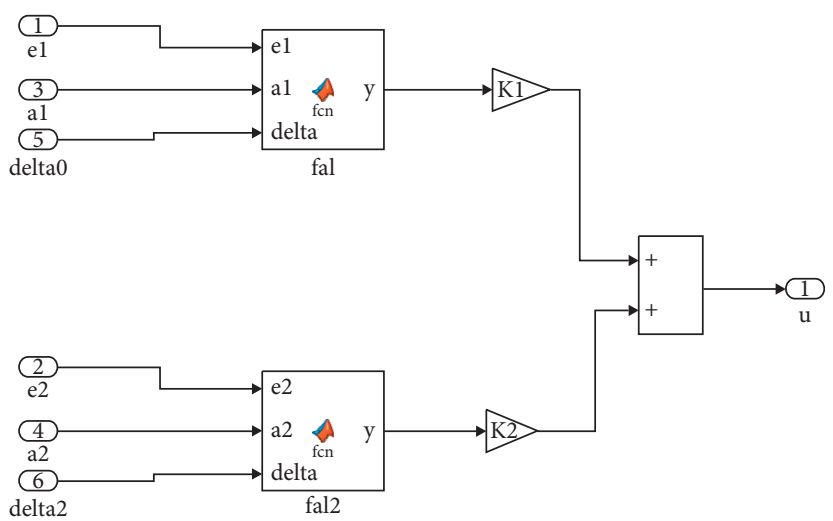

FIGURE 11: NLSEF simulation model.

In this test, the simulation time is $35 \mathrm{~s}$. The synchronous generators G1 and G2 start running in parallel with no load at $0 \mathrm{~s}$, are suddenly loaded with Load 1 at $5 \mathrm{~s}$, are suddenly loaded with Load 2 at $15 \mathrm{~s}$, and are suddenly unloaded of Load 2 at $25 \mathrm{~s}$. The load parameter settings of Load 1 and Load 2 are $P=1.5 \times 10^{3} \mathrm{~kW}$ and $Q=1.2 \times 10^{3} \mathrm{~kW}$. The curves of the changes in each parameter of the synchronous generator are shown in Figure 16.

Figure 16 shows that when the synchronous generator starts with no load at $0 \mathrm{~s}$, in the cases where the excitation system uses the control methods of AVR and AVR + PSS2B, the generator can quickly start, and in the case where ADRC is used, $U_{t}$ has an adjustment process with a large amplitude, which shows that, under no disturbances, the regulation effect of AVR and AVR + PSS2B is better than that of ADRC. With the sudden loading at $5 \mathrm{~s}$ and $15 \mathrm{~s}$, the use of ADRC for approximately $0.3 \mathrm{~s}$ can restore $U_{\mathrm{t}}$ to a stable value, and almost no overshooting occurs in the regulation process, whereas the regulation time using AVR + PSS2B is approximately $1 \mathrm{~s}$, and the voltage slightly overshoots in the regulation process. In the case where only AVR is used, the amount of overshooting in voltage is the largest, and the regulation time is the longest. With the sudden unloading at $25 \mathrm{~s}$, after the maximum voltage is increased to 1.02 with the use of ADRC and the voltage is restored to 1 after $0.2 \mathrm{~s}$, the maximum voltage is increased to 1.05 with the use of AVR + PSS2B and AVR, the voltage tends to stabilize after approximately $3 \mathrm{~s} 23$, and the voltage fluctuation is smaller in the AVR + PSS2B regulation process.

Figure 17 shows that, with the sudden loading at $5 \mathrm{~s}$ and $15 \mathrm{~s}$ as well as the sudden unloading at $25 \mathrm{~s}$, in the case where the excitation system is using AVR control, the fluctuation in the rotational speed of the synchronous generator is the greatest, and the regulation time is the longest; when AVR + PSS2B is used, the maximum value and the minimum value of the rotational speed fluctuation are both reduced, 


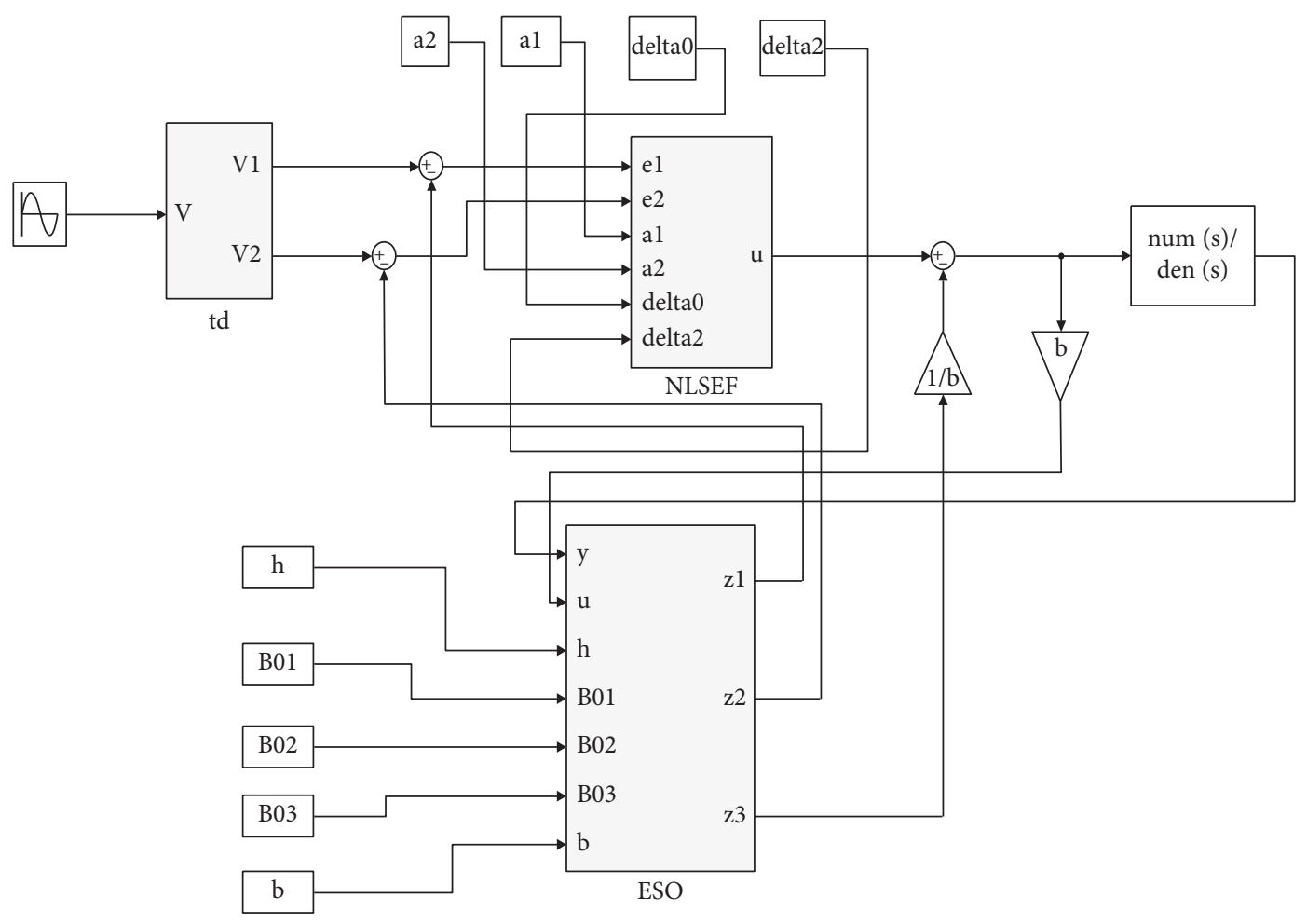

FIGURE 12: ADRC controller simulation model.

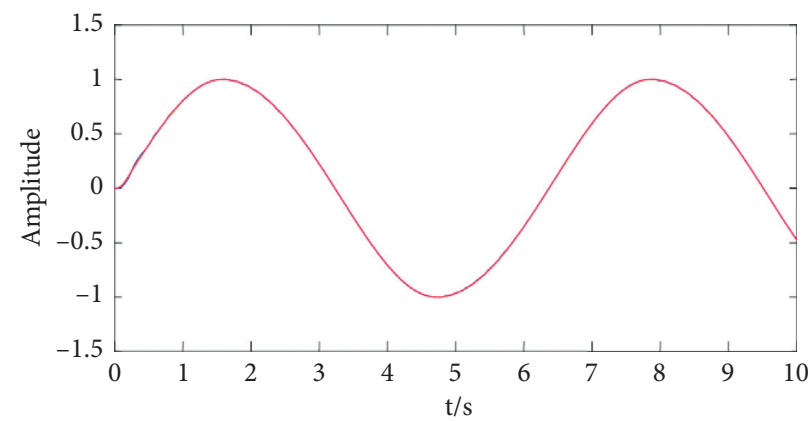

$-\mathrm{Z} 1$

- X1

FIgURE 13: State $(\mathrm{x})_{1}$ estimation curve.

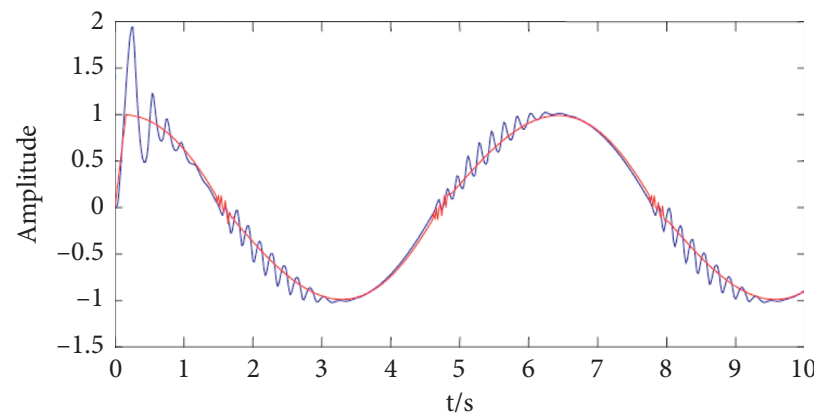

$-\mathrm{Z} 2$

$-\mathrm{X} 2$

Figure 14: State $(\mathrm{x})_{2}$ estimation curve. 


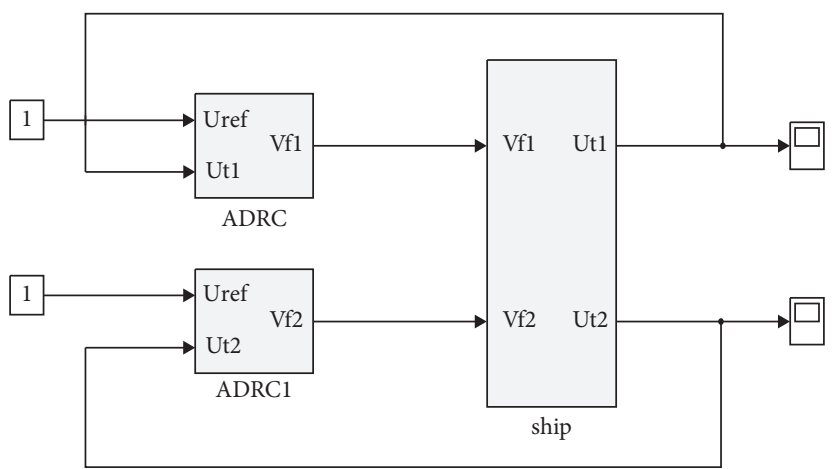

FIGURE 15: ADRC-based two-generator parallel-running simulation model of a ship power system.

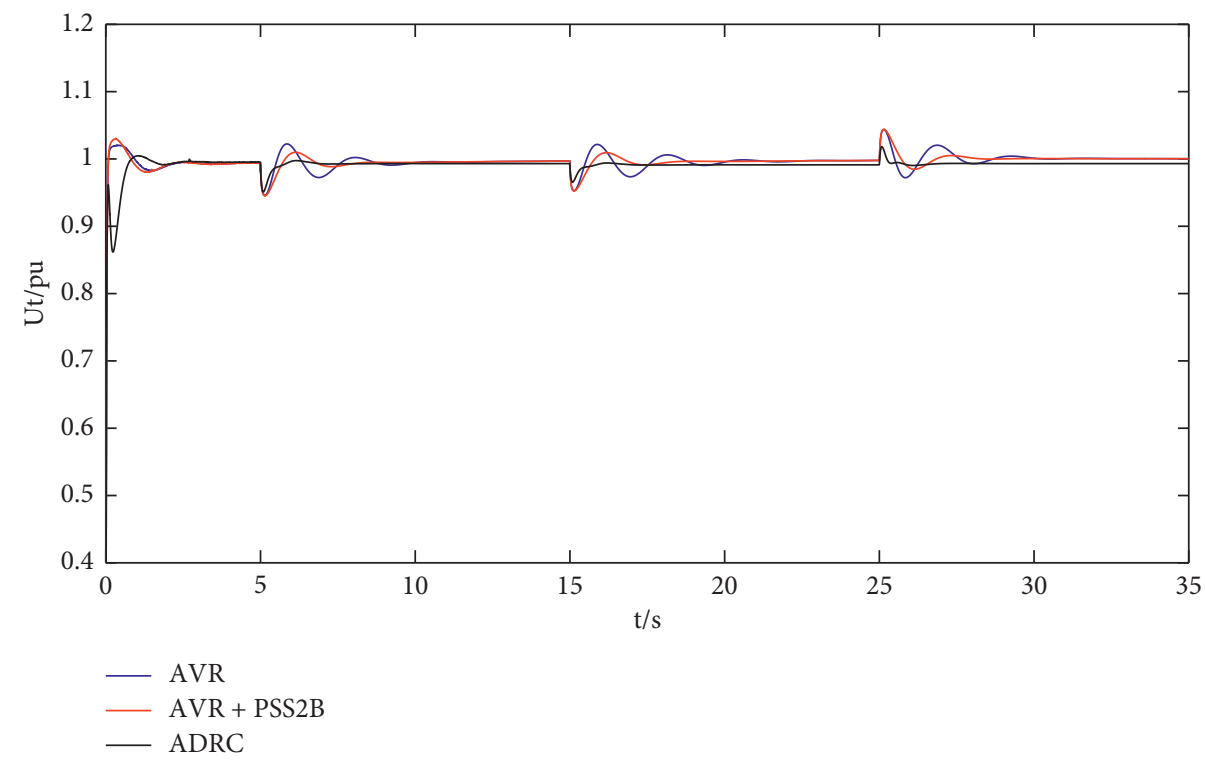

FIGURE 16: Comparison of the terminal voltages of the synchronous generator under sudden loading and sudden unloading working conditions.

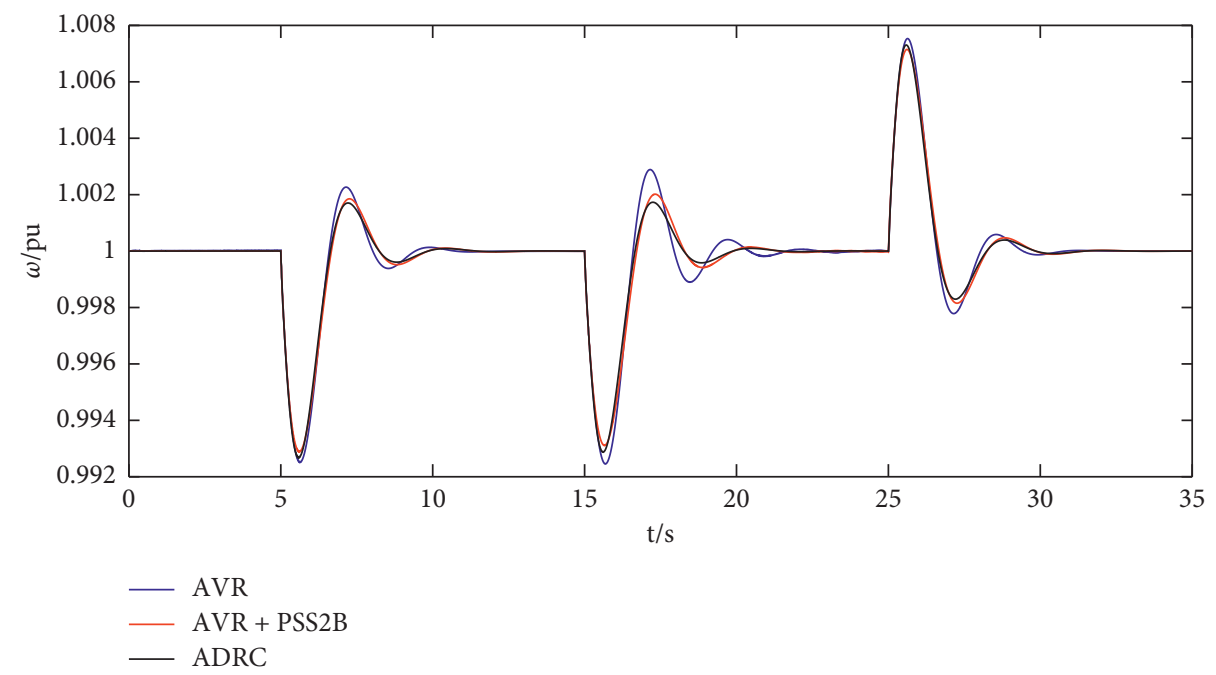

FIGURE 17: Comparison of the rotational speeds of the synchronous generator under sudden loading and sudden unloading working conditions. 
and the time needed for restoration to a stable value is shorter. After ADRC is used, the rotational speed fluctuation of the synchronous generator reaches the minimum.

As seen in Figure 18, after sudden loading with Load 1 at $5 \mathrm{~s}$, the active power is increased to 0.2 , and after sudden loading with Load 2 at $15 \mathrm{~s}$, the active power is increased to two times the original value. Under the two control modes of AVR and AVR + PSS2B, after the active power is restored to a stable value, there is a slight fluctuation, and after ADRC is used, the active power exhibits a large fluctuation only for an instant at sudden loading; afterwards, this parameter is quickly restored to the stable value and does not oscillate after restoration to the stable value. At $25 \mathrm{~s}$, when half of the load is suddenly unloaded, after a slight fluctuation, the active power drops to half of the original, and the ADRC restores the steady-state value the fastest.

Figure 19 shows that the mechanical power of the synchronous generator running with no load from 0 to $5 \mathrm{~s}$ is 0 , the mechanical power increases after sudden loading at $5 \mathrm{~s}$ and $15 \mathrm{~s}$, and the mechanical power drops to half of the original after the sudden unloading of half the load at $25 \mathrm{~s}$. In this process, under the three cases of AVR, AVR + PSS2B, and $\mathrm{ADRC}$, the ADRC regulation time is the shortest, with the smallest amplitude fluctuation in the active power followed by AVR + PSS2B, and the AVR regulation time is the longest, with the largest amplitude fluctuation in the active power.

The above simulation results show that, under sudden loading and sudden unloading working conditions for the ship power system, compared with the control methods of AVR and AVR + PSS2B, when the excitation system uses the ADRC method, the oscillation amplitudes of the parameters of the synchronous generator are the smallest, and the time of restoration to stability is the fastest.

4.3. Sudden Loading and Single-Phase Short-Circuit Fault Simulation Test. In this test, the simulation time is $25 \mathrm{~s}$, the synchronous generators G1 and G2 start running parallelly with no load at $0 \mathrm{~s}$, the heavy load of Load 3 is suddenly added at $5 \mathrm{~s}$, and a single-phase short-circuit fault occurs suddenly at $15 \mathrm{~s}$, with a fault duration of $0.1 \mathrm{~s}$. The load parameter settings of Load 3 are $P=3.1 \times 10^{3} \mathrm{~kW}$ and $Q=2.5 \times 10^{3}$ kvar. The curves of the changes of each parameter of the synchronous generator are shown in Figure 20.

As seen from Figure 20, when a heavier load is suddenly loaded at $5 \mathrm{~s}$, the results are still the same as the analysis of the previous scenario; the control effect of the ADRC is the best, followed by that of AVR + PSS2B, and the control effect of AVR is the worst. When a single-phase short-circuit fault occurs at $15 \mathrm{~s}$, an obvious drop appears in the terminal voltage of the synchronous generator under the three control modes of AVR, AVR + PSS2B, and ADRC, and the amplitudes of the drops are basically the same; however, under the two control modes of AVR and AVR + PSS2B, the voltage needs to undergo approximately $2.5 \mathrm{~s}$ of regulation before it is restored to stability, and the voltage oscillation amplitude in the AVR regulation process is greater than that of
AVR + PSS2B. Under ADRC, the voltage needs to go through only approximately $1 \mathrm{~s}$ of restoration, it can be restored to the stable value, and the voltage oscillation amplitude in the regulation process is very small.

Figure 21 shows that when the heavy load is suddenly added at $5 \mathrm{~s}$, the differences in the curves of the rotational speed for the three control modes are relatively large, with the control effect of ADRC being the best; when the shortcircuit fault suddenly occurs at $15 \mathrm{~s}$, a slight drop appears in the rotational speed of the synchronous generator, dropping to 0.996 under all three control modes, and then the voltage is restored to a stable value after undergoing 3-4 s [23] of regulation. In the regulation process, the oscillation in the rotational speed of the AVR control mode is somewhat large, and the curves for the two control methods of AVR + PSS2B and ADRC are almost the same.

Figure 22 shows that when the heavy load is suddenly added at $5 \mathrm{~s}$, the active power increases to 0.5 after undergoing transient oscillation; the oscillation amplitude with ADRC is the smallest, and its time for restoration to a steady state is the shortest. The control effect of AVR + PSS2B is the second best, followed by that of AVR. When the single-phase short-circuit fault occurs at $15 \mathrm{~s}$, a sudden change occurs in the active power, which increases to 1.4 at the maximum and drops to 0.3 at the minimum; the curves of the three control modes are almost the same.

Figure 23 shows that when the heavy load is suddenly added at $5 \mathrm{~s}$, the mechanical power quickly rises and stabilizes at 0.45 after undergoing transient regulation. The oscillation amplitude is the greatest under the AVR control mode, and the increase in the oscillation amplitude is the smallest with ADRC. After the short-circuit fault occurs at $15 \mathrm{~s}$, a slight sudden increase occurs in the mechanical power, which is restored to stability after undergoing approximately $3 \mathrm{~s}$ of regulation; the effects of the three control modes are almost the same.

The above analysis shows that when small disturbances such as single-phase short-circuit faults occur in the ship power system, the restraining effect of ADRC on the oscillation of various parameters of the synchronous generator is slightly better than those of the other two control methods.

4.4. Three-phase Short-Circuit Fault Simulation Test. In this test, the simulation time setting is $20 \mathrm{~s}$. The synchronous generators G1 and G2 start at $0 \mathrm{~s}$ with Load 3, and a threephase short-circuit fault occurs in the system at $10 \mathrm{~s}$, with a fault duration of $0.1 \mathrm{~s}$. The curves of the changes in each parameter of the synchronous generator are shown in Figure 24.

As seen from Figure 24, when the three-phase shortcircuit fault occurs in the system at $10 \mathrm{~s}$, a large drop occurs in the terminal voltage of the synchronous generator, and then the terminal voltage quickly rallies and is restored to stability. Under AVR control, the terminal voltage drops to a minimum of 0.2 , increases to a maximum of 1.185 , and is restored to stability after approximately $5 \mathrm{~s}$. Under AVR + PSS2B control, the terminal voltage drops to a minimum of 0.2 , increases to a maximum of 1.18 , and is 


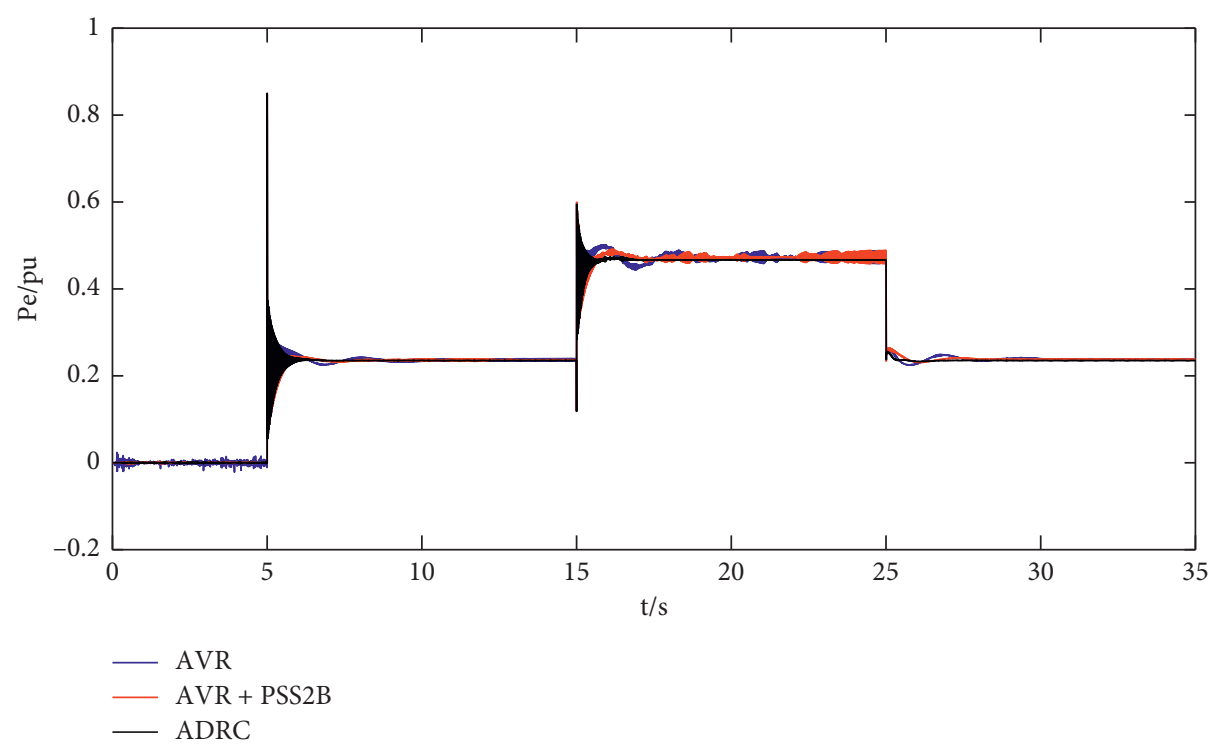

FIGURE 18: Comparison of the active powers of the synchronous generator under sudden loading and sudden unloading working conditions.

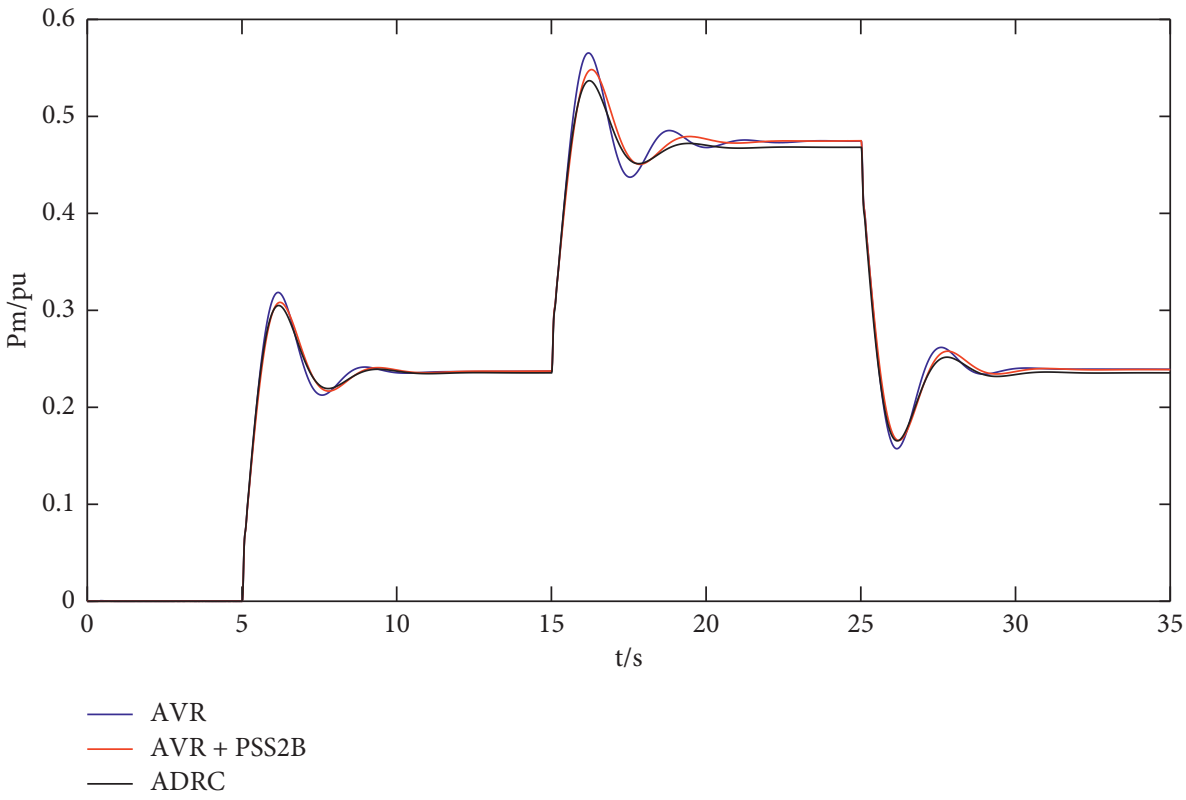

FIGURE 19: Comparison of the mechanical powers of the synchronous generator under sudden loading and sudden unloading working conditions.

restored to stability after approximately $3 \mathrm{~s}$. Under ADRC, the terminal voltage drops to a minimum of 0.2 , increases to a maximum of 1.15 , and is restored to stability after approximately $2 \mathrm{~s}$.

Figure 25 shows that after the three-phase short-circuit fault occurs in the system at $10 \mathrm{~s}$, an obvious drop appears in the rotational speed of the synchronous generator, and the rotational speed is restored to stability after undergoing a short period of oscillation regulation. Under AVR control, the rotational speed drops to a minimum of 0.987 , increases to a maximum of 1.009 , and is restored to stability after undergoing approximately $6 \mathrm{~s}$ of regulation. Under
AVR + PSS2B control, the rotational speed drops to a minimum of 0.987 , increases to a maximum of 1.007 , and is restored to stability after undergoing approximately $5 \mathrm{~s}$ of regulation. Under ADRC, the rotational speed drops to a minimum of 0.987 , increases to a maximum of 1.005 , and is restored to stability after undergoing approximately $4 \mathrm{~s}$ of regulation.

Figure 26 shows that after the three-phase short-circuit fault occurs in the system at $10 \mathrm{~s}$, a large oscillation appears instantaneously in the active power of the synchronous generator, and the active power is restored to stability after undergoing a short period of oscillation regulation. Under 


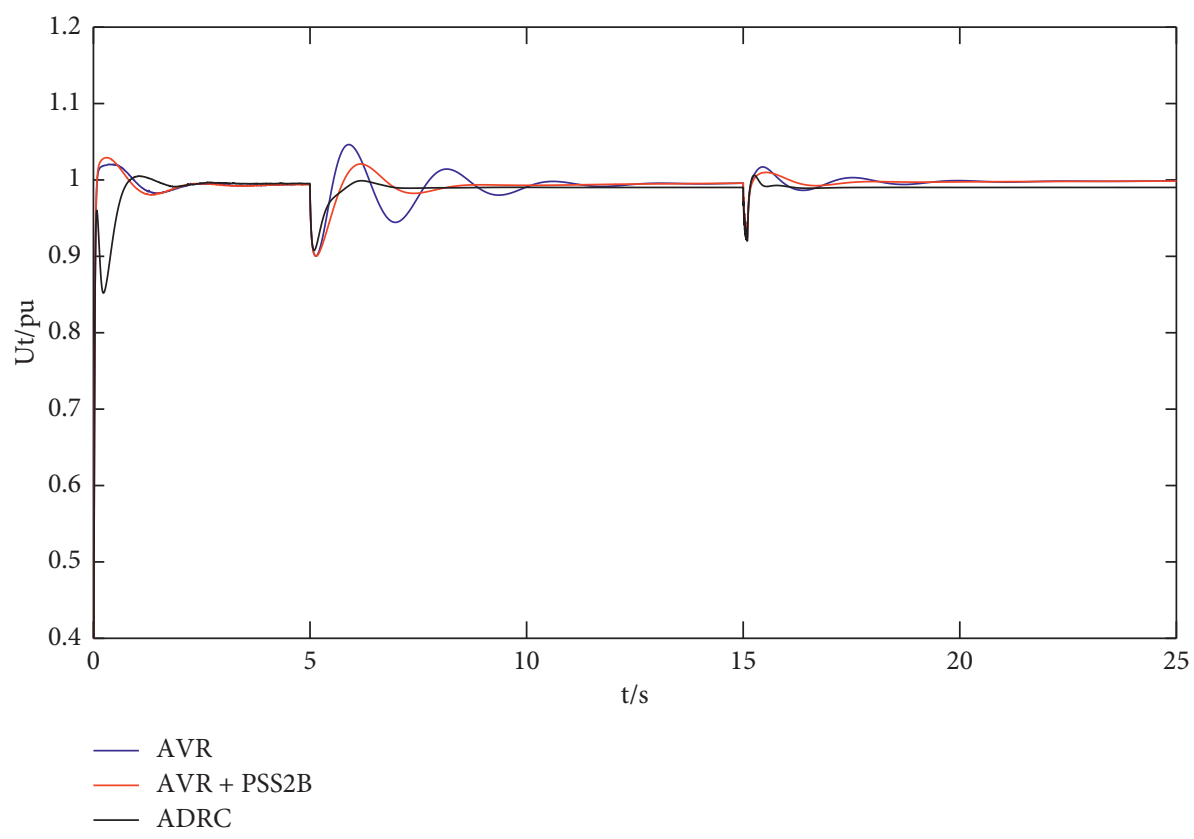

FIGURE 20: Comparison of the terminal voltages of the synchronous generator under sudden loading and single-phase short-circuit fault working conditions.

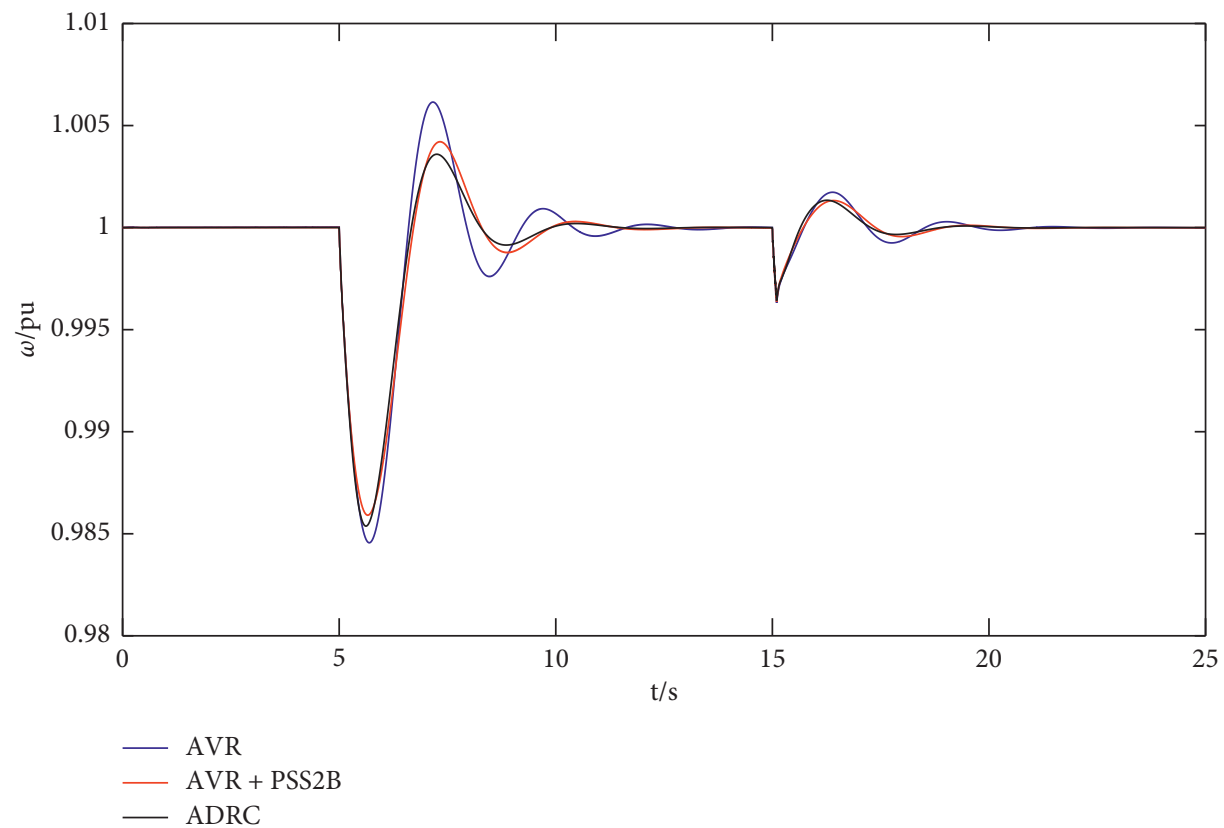

FIGURE 21: Comparison of the rotational speeds of the synchronous generator under sudden loading and single-phase short-circuit fault working conditions.

AVR control, the active power drops to a minimum of 0 , increases to a maximum of 3.3, and is restored to stability after undergoing approximately $2.5 \mathrm{~s}$ of regulation. Under AVR + PSS2B control, the active power drops to a minimum of 0 , increases to a maximum of 3.3 , and is restored to stability after undergoing approximately $2.2 \mathrm{~s}$ of regulation. Under ADRC, the active power drops to a minimum of 0 , increases to a maximum of 3.2, and is restored to stability after undergoing approximately $1.5 \mathrm{~s}$ of regulation.

Figure 27 shows that after the three-phase short-circuit fault occurs in the system at $10 \mathrm{~s}$, the mechanical power of the synchronous generator increases instantaneously, falls back, and is restored to stability after undergoing a short period of oscillation regulation. Under AVR control, the 


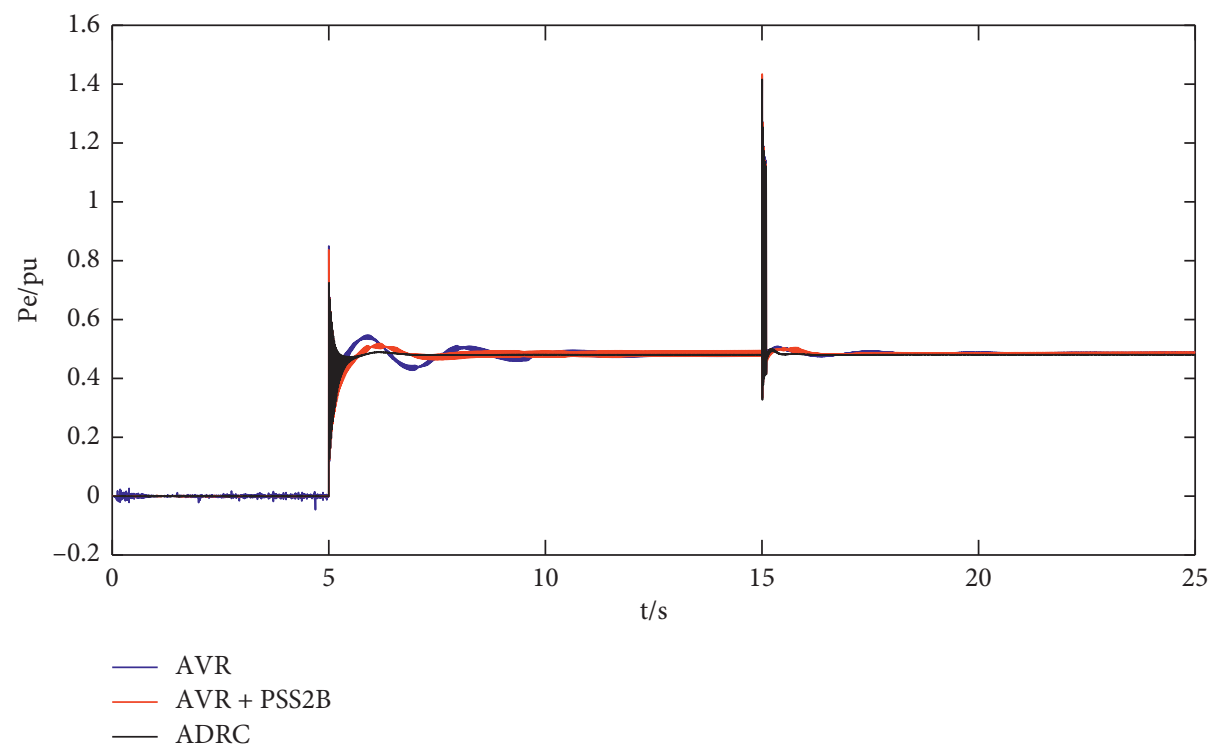

FIGURE 22: Comparison of the active powers of the synchronous generator under sudden loading and single-phase short-circuit fault working conditions.

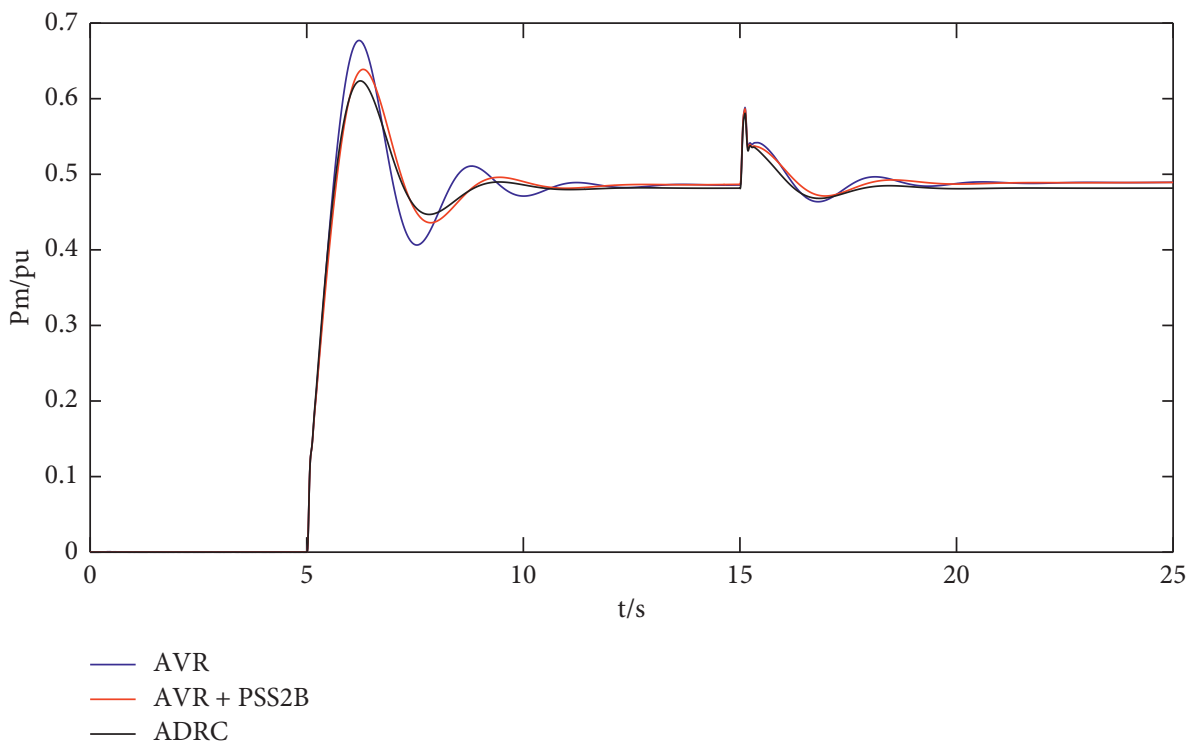

FIGURE 23: Comparison of the mechanical powers of the synchronous generator under sudden loading and single-phase short-circuit fault working conditions.

mechanical power drops to a minimum of 0.35 , increases to a maximum of 0.88 , and is restored to stability after undergoing approximately $6 \mathrm{~s}$ of regulation. Under AVR + PSS2B control, the mechanical power drops to a minimum of 0.37 , increases to a maximum of 0.88 , and is restored to stability after undergoing approximately $4.5 \mathrm{~s}$ of regulation. Under ADRC, the mechanical power drops to a minimum of 0.4 , increases to a maximum of 0.88 , and is restored to stability after undergoing approximately $3 \mathrm{~s}$ of regulation.

The above simulation analysis in this section shows that when larger disturbances such as three-phase short-circuit faults occur in the ship power system, compared with the control methods of AVR and AVR+PSS2B, the ADRC 


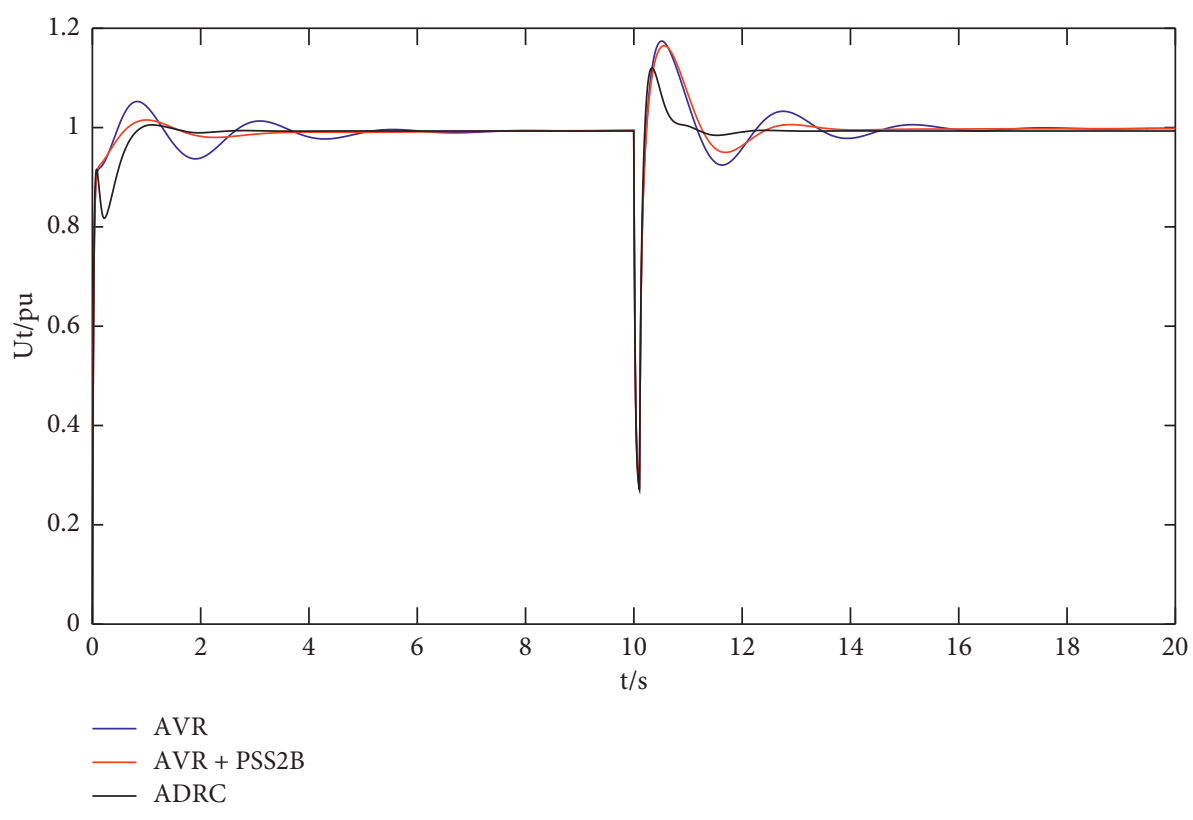

FIGURE 24: Comparison of the terminal voltages of the synchronous generator under three-phase short-circuit fault working condition.

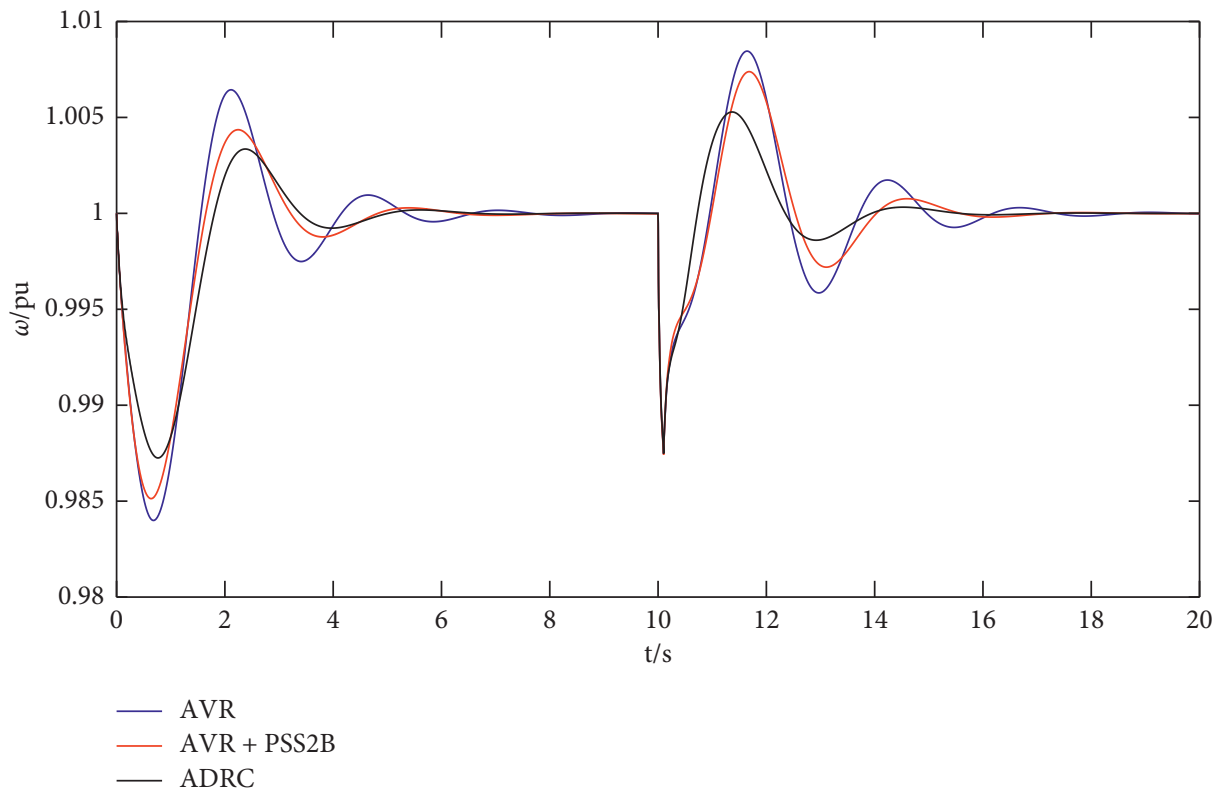

FIGURE 25: Curves comparing the rotational speed of the synchronous generator under three-phase short-circuit fault working conditions. 


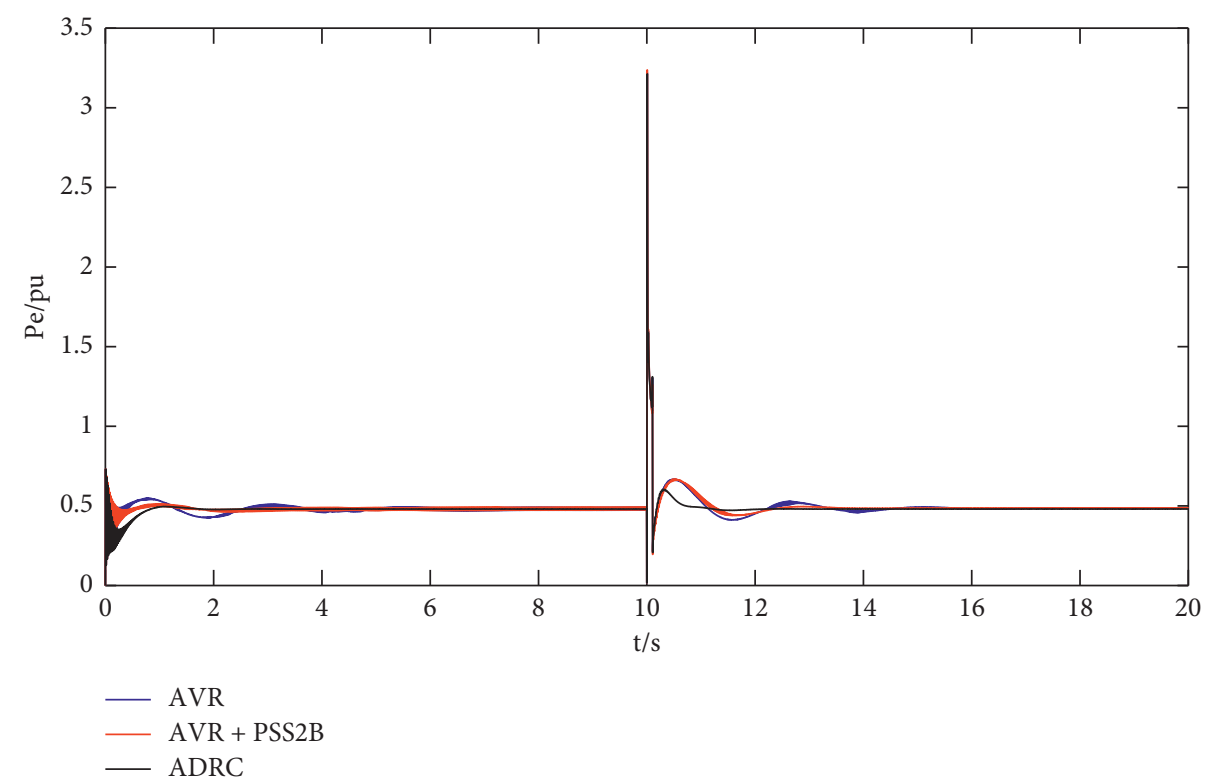

FIGURE 26: Comparison of the active powers of the synchronous generator under three-phase short-circuit fault working conditions.

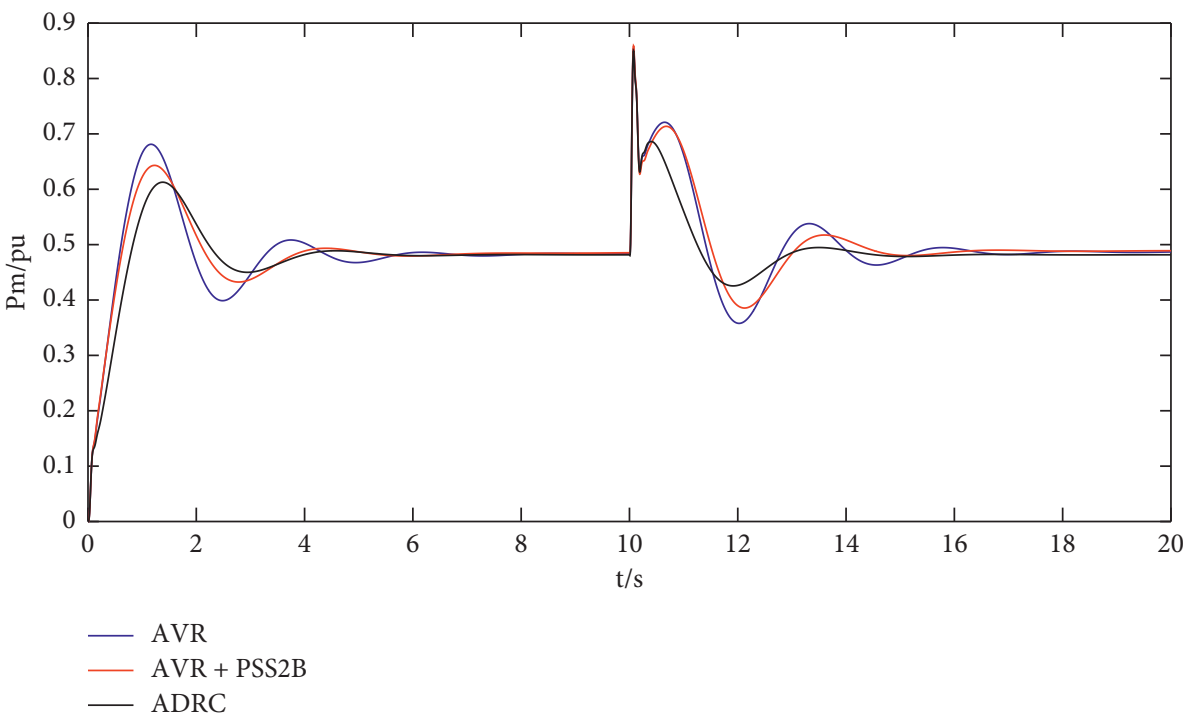

FIGURE 27: Comparison of the mechanical powers of the synchronous generator under three-phase short-circuit fault working conditions.

method has a better restraining and regulating effect on synchronous generator oscillations caused by large disturbances.

\section{Conclusion}

In this paper, the negative damping effect of the excitation system is first analyzed from the perspective of synchronous torque and damping torque in accordance with the HeffronPhillips model of the synchronous generator, and the application of ADRC to the synchronous generator excitation system of a ship is proposed. Finally, simulation experiments of the two-generator parallel-running system of the synchronous generator of a ship are carried out under different working conditions, comparative simulation tests are carried out under different working conditions for the three excitation control methods of AVR, AVR + PSS2B, and ADRC, and the simulation results show the effectiveness of the proposed method. Notably, when the system is subjected to disturbances under different working conditions, the restraining effect of $\mathrm{ADRC}$ on the oscillations of various parameters of the synchronous generator is the best. ADRC replaces the PID control in the traditional marine synchronous generator, and constructs an ADRC-based marine 
synchronous generator excitation control system in the future.

\section{Data Availability}

The data used to support the findings of this study are available from the corresponding author upon request.

\section{Conflicts of Interest}

The authors declare that they have no conflicts of interest.

\section{Acknowledgments}

This work was supported in part by the National Natural Science Foundation of China under Grant nos. 51879118 and 51779102, the High-Level Talent Training Project in the Transportation Industry under Grant no. 2019-014, the Fujian Province Office of Science and Technology Support for Army under Grant no. B19101, the Foundation of Fujian Education Committee of China for New Century Distinguished Scholars under Grant no. B17159, the Scientific Research Foundation of Key Laboratory of Fishery Equipment and Engineering, Ministry of Agriculture of the People's Republic of China under Grant nos. 2016002 and 2018001, the Scientific Research Foundation of Artificial Intelligence Key Laboratory of Sichuan Province under Grant no. 2017RYJ02, and the Scientific Research Foundation of Jiangsu Key Laboratory of Power Transmission \& Distribution Equipment Technology under Grant no. 2017JSSPD01.

\section{References}

[1] T. H. Tang, Ship Electric Propulsion System, China Machine Press, Beijing, China, 2015.

[2] R. J. Wang, Y. J. Zhan, and H. F. Zhou, "Research on optimal sizing of stand-alone diesel-based hybrid energy systems," Acta Energiae Solaris Sinica, vol. 40, no. 2, pp. 348-355, 2019.

[3] H. Jun, J. Sun, and H. Hofmann, "Mitigating power fluctuations in electric ship propulsion with hybrid energy storage system: design and analysis," IEEE Journal of Oceanic Engineering, vol. 43, no. 1, pp. 93-107, 2018.

[4] S. Lalit-Chandra, "Performance of coordinated interline power flow controller and power system stabilizer in combined multiarea restructured ALFC and AVR system," International Transactions on Electrical Energy Systems, vol. 29, no. 3, pp. 2822-2839, 2019.

[5] H. Reza, "Power system stabilizer design based on optimal model reference adaptive system," Ain Shams Engineering Journal, vol. 9, no. 2, pp. 311-318, 2016.

[6] M. Luan, W. Lu, and D. C. Liu, "Coordination excitation controller design based on disturbance tracking Terminal sliding mode and multi-objective zero dynamics control," Power System Protect and Control, vol. 43, no. 4, pp. 311-318, 2015.

[7] Y. Jiang and Z.-P. Jiang, "Robust adaptive dynamic programming for large-scale systems with an application to multimachine power systems," IEEE Transactions on Circuits and Systems II: Express Briefs, vol. 59, no. 10, pp. 693-697, 2012.

[8] B. J. Lei, S. M. Fei, and J. Y. Zhai, "Coordinated control of automatic voltage regulator and SVC in multi-machine power system," Transactions of China Electrotechnical Society, vol. 30, no. 18, pp. 131-139, 2015.

[9] X. W. Zhan, H. Xie, and S. X. Lv, "Parameter setting and onsite test of power system stabilizer -PSS4B," Power System Technology, vol. 40, no. 2, pp. 508-513, 2016.

[10] H. Wang, W. Wei, and Y. F. Pan, "A bilevel programming based method for tuning parameters of nonlinear robust power system stabilizer," Automation of Electric Power System, vol. 38, no. 14, pp. 42-48, 2014.

[11] Z. Ilias and X. Wang, "Wide-Area damping control for interarea oscillations in power grids based on PMU measurements," IEEE Control Systems Letters, vol. 2, no. 4, pp. 719-724, 2018.

[12] X. Y. Liu, The Research on Methods of Suppressing Oscillation of Synchronous Generator in Ship Power System, Jimei University, Xiamen, China, 2020.

[13] C. Yan, G. K. Venayagamoorthy, and K. Corzine, "AIS-based coordinated and adaptive control of generator excitation systems for an electric ship," IEEE Transactions on Industrial Electronics, vol. 59, no. 8, pp. 3102-3112, 2012.

[14] H. Shayeghi and Y. Hashemi, "Profit enhancement by a set of performance and robustness indices based design of dualdimensional PODC and PSS2B in smart grids," Energy Conversion and Management, vol. 83, no. 7, pp. 99-109, 2014.

[15] H. Shayeghi and Y. Hashemi, "Pecuniary evaluation of provided service by local and global based dual-dimensional SDC and PSS2B in the context of deregulated power markets," Energy Conversion and Management, vol. 76, no. 7, pp. 753-763, 2013.

[16] G. Sulligoi, A. Vicenzutti, V. Arcidiacono, and Y. Khersonsky, "Voltage stability in large marine-integrated electrical and electronic power systems," IEEE Transactions on Industry Applications, vol. 52, no. 4, pp. 3584-3594, 2016.

[17] Q. Sun, R. Han, H. Zhang, J. Zhou, and J. M. Guerrero, "A multiagent-based consensus algorithm for distributed coordinated control of distributed generators in the energy internet," IEEE Transactions on Smart Grid, vol. 6, no. 6, pp. 3006-3019, 2015.

[18] R. Wang, Q. Sun, W. Hu, Y. Li, D. Ma, and P. Wang, "SoCbased droop coefficients stability region analysis of the battery for stand-alone supply systems with constant power loads," IEEE Transactions on Power Electronics, vol. 36, no. 7, pp. 7866-7879, 2021.

[19] W. Hu, C. Ruan, H. Nian, and D. Sun, "Zero-sequence current suppression strategy with common mode voltage control for open-end winding PMSM drives with common DC bus," IEEE Transactions on Industrial Electronics, vol. 68, no. 6, pp. 4691-4702, 2020.

[20] D. Ma, X. Hu, H. Zhang, Q. Sun, and X. Xie, "A hierarchical event detection method based on spectral theory of multidimensional matrix for power system," IEEE Transactions on Systems, Man, and Cybernetics: Systems, vol. 51, no. 4, pp. 2173-2186, 2019.

[21] X. Hu, H. Zhang, D. Ma, and R. Wang, "A tnGAN-based leak detection method for pipeline network considering incomplete sensor data," IEEE Transactions on Instrumentation and Measurement, vol. 70, pp. 1-10, 2020.

[22] J. Q. Han, "Auto disturbances rejection control technique," Frontier Science, vol. 98, no. 1, pp. 24-31, 2007.

[23] B. Payvand and S. M. H. Hosseini, "A new method for mitigating frequency fluctuations in ships with electrical propulsion," ISA Transactions, vol. 92, no. 1, pp. 241-256, 2019. 